\title{
Poly(butylene 2,4-furanoate), an Added Member to the Class of Smart Furan-Based Polyesters for Sustainable Packaging: Structural Isomerism as a Key to Tune the Final Properties
}

\author{
Enrico Bianchi, Michelina Soccio, Valentina Siracusa, Massimo Gazzano, Shanmugam Thiyagarajan,* \\ and Nadia Lotti*
}

Cite This: ACS Sustainable Chem. Eng. 2021, 9, 11937-11949

Read Online

ABSTRACT: High-molecular-weight poly(butylene 2,4-furanoate) (2,4-PBF), an isomer of well-known poly(butylene 2,5furanoate) (2,5-PBF), was synthesized through an eco-friendly solvent-free polycondensation process and processed in the form of an amorphous film by compression molding. Molecular characterization was carried out by NMR spectroscopy and GPC analysis, confirming the chemical structure and high polymerization degree. Thermal analyses evidenced a reduction of both glass-to-rubber transition and melting temperatures, as well as a detriment of crystallization capability, for 2,4-PBF with respect to 2,5-PBF. Nevertheless, it was possible to induce crystal phase formation by annealing treatment. Wide-angle X-ray scattering revealed that the crystal lattices developed in the two isomers are distinct from each other. The different isomerism affects also the thermal stability, being 2,4-PBF more thermally inert than 2,5-PBF. Functional properties, such as wettability, mechanical response, and gas barrier capability, were tested on both amorphous and semicrystalline 2,4-PBF films and compared with those of 2,5-PBF. Reduced hydrophilicity was determined for 2,4-isomer, in line with its lower average dipole moment, suggesting better chemical resistance to hydrolysis. Stress-strain tests have evidenced the higher flexibility and toughness of 2,4-PBF with respect to those of 2,5-PBF and the possibility of improving its mechanical resistance by annealing. Finally, the different isomerism deeply affects the gas barrier performance, being the $\mathrm{O}_{2}$ - and $\mathrm{CO}_{2}$-transmission rates of 2,4-PBF 50 and 110 times lower, respectively, than those of 2,5-PBF. The gas barrier properties turned out to be outstanding under a dry atmosphere as well as in humid conditions, suggesting the presence of interchain hydrogen bonds. The gas blocking capability decreases after annealing because of the presence of disclination associated with the formation of crystals.

KEYWORDS: 2,4-furandicarboxylic acid, 2,5-furandicarboxylic acid, structural isomerism, thermal properties, diffractometric analysis, mechanical properties, gas barrier properties

\section{INTRODUCTION}

No one can dispute the fact that the use of plastics is inevitable in modern society. In fact, compared to metallic and nonmetallic materials, plastics possess a whole plethora of properties (such as availability, lightweight, easy processing, economy, colorability, acoustic, thermal, electrical insulation, smart mechanical response, resistance to corrosion and chemical inertia, as well as water repellency and unassailable by molds, fungi, and bacteria, and many more) that justify their extensive use in the most varied fields (such as packaging, healthcare, fisheries, and agriculture, to cite only a few).

Although the advantages of plastics are innumerable and important, their massive production from virgin monomers and the inefficient waste management by their end of life have generated a substantial environmental impact, which unfortunately affects both the terrestrial and marine habitats. ${ }^{1,2}$ Therefore, mismanagement of plastic waste undermines the ability of the global community to achieve the carbon emission targets necessary to fight climate change (United Nations Sustainable Development Goals 7 and 13; UN, 2013).

To reduce such a huge environmental impact of plastics and their dispersion into the environment; local, national, and international political institutions have introduced several

Received: June 20, 2021

Revised: $\quad$ August 7, 2021

Published: August 21, 2021 
directives, which include taxes and legislative bans, such as that related to single-use plastics. ${ }^{3,4}$

The long-term goals of transitioning toward circularity of plastics and sustainable economy presuppose recycling as the best option for efficient management of plastic waste. Both chemical and mechanical recycling methods have their own merits of transforming the plastic waste further into (new) products; the former gives an opportunity to use (consume) spent plastics as a feedstock to derive primary raw materials (i.e., monomers), which can be used to produce virgin plastics (i.e., circularity). The increasing interest in developing and establishing efficient and cost-effective recycling techniques for the used plastics (single- and multilayer materials) is clearly evident from the number of publications and patents. ${ }^{5,6}$

The alternative solution lies in the eco-design of polymers from renewable sources (potentially from agrifood waste). ${ }^{7-16}$ Furan-based polyesters represent a class of bioplastics with enormous potential. 2,5-Furandicarboxylic acid (2,5-FDCA) is widely advocated as a renewable alternative monomer to fossil-derived p-terephthalic acid (PTA) in polyester synthesis. Many studies have shown that poly(ethylene 2,5-furanoate) (PEF), the polyester synthesized from 2,5-FDCA and ethylene glycol (EG), has superior properties (thermal, mechanical, and gas barrier) compared to its terephthalate analogue poly(ethylene terephthalate) (PET). It is worth mentioning here that these superior properties have significant advantages especially in packaging applications that meet the demands like high heat resistance and lower melting temperature, making the blow molding and extrusion process easier. ${ }^{14,17-23}$

The outstanding performances of PEF polymers have received considerable attention from various chemicals companies. This resulted in the transition from the labscale synthesis to the industrial production of 2,5-FDCA predominantly from carbohydrate feedstocks. ${ }^{24}$ The front runners in producing this monomer include the following: Avantium Chemicals (pilot plant, 40 tonnes/year), DuPont and ADM (pilot plant, 60 tonnes/year), AVA Biochem (production, $30 \mathrm{kt} /$ year), and Corbion. ${ }^{25}$

In addition to EG, a wide range of longer flexible aliphatic glycols were used to prepare various random and block furanbased copolymers later on. ${ }^{26-28}$ For example, poly(propylene 2,5-furanoate) (PPF) and poly(butylene 2,5-furanoate) (PBF), derived from 1,3-propanediol (1,3-PDO) and 1,4butanediol (1,4-BDO), respectively, hold thermal and mechanical properties comparable to commercial terephthalate analogues (PPT and PBT). ${ }^{29-32,34-36,38-43,45-48}$ Some specific works aimed to evaluate the possible use of these new biobased materials in food packaging by investigating the functional properties (mechanical and barrier) of the corresponding films obtained by compression molding. ${ }^{7-27}$

Besides 2,5-FDCA, another isomer, i.e., 2,4-furandicarboxylic acid (2,4-FDCA), is also a potentially interesting monomer in polyester synthesis, which has been overlooked in the last decades. 2,4-FDCA is formed up to $30 \%$ in a onepot Henkel-type disproportionation reaction, starting from potassium-2-furoate (based on agricultural residues), together with 2,5-furandicarboxylic acid (2,5-FDCA) (main product) and 3,4-furandicarbocxylic acid (3,4-FDCA) $(<5 \%){ }^{49,50} 2,4-$ FDCA is an unsymmetrical molecule containing two carboxylic acid groups in $\mathrm{C} 2$ and $\mathrm{C} 4$ positions, whereas in 2,5-FDCA at $\mathrm{C} 2$ and $\mathrm{C} 5$ positions. Thiyagarajan and co- workers have investigated the structural characteristics of dimethyl esters of 2,4-FDCA through single-crystal X-ray diffraction studies; the interatomic distance between the carboxylic acid groups in 2,4-FDCA is $5.075 \AA$, while that in 2,5-FDCA is $4.830 \AA$, and the projected angle between the two substituents is larger for $2,4-\mathrm{FDCA}\left(150^{\circ}\right.$ vs $129^{\circ}$ for 2,5-FDCA). The position of the two carboxyl substituents in 2,4-FDCA makes this isomer less symmetric than 2,5-FDCA, which explains the lower ability of 2,4-PEF to crystallize compared to 2,5-PEF. ${ }^{51}$

Polyesters from 2,4-FDCA and their properties are still less studied, the most investigated being poly(ethylene 2,4furandicarboxylate) (2,4-PEF). Bourdet et al., using dielectric relaxation spectroscopy, reported that the $\beta$ transition activation energy of 2,4-PEF was higher compared to that of 2,5-PEF ( 75 vs $56 \mathrm{~kJ} / \mathrm{mol}$ ); moreover, the average dipole moment of 2,4-PEF polymer chains was considerably lower than that of 2,5 -PEF (6.2 vs $8.2 \mathrm{D}$, respectively). ${ }^{52,53}$ Nolasco et al., through a combination of vibrational spectroscopy techniques (infrared, Raman, and inelastic neutron scattering) and $\mathrm{ab}$ initio calculations, have assessed that 2,4-PEF randomly coiled chains based on gauche-ethylene glycol segments are favored. ${ }^{54}$

In addition to EG, only one report is available to date describing the synthesis and physical characterization of polyesters prepared from other linear glycols (1,3-PDO and $1,4-\mathrm{BDO})$ in combination with 2,4-FDCA. ${ }^{50}$ Very recently, the copolymers synthesized using different ratios of 2,4FDCA in combination with 2,5-FDCA and PTA were investigated. $^{53,55}$

To the best of our knowledge, this is the first report dealing with the potential of 2,4-FDCA-based polyesters in the food packaging sector by studying their processability to form films, of which to analyze the mechanical behavior as well as the gas barrier performance.

In the present study, the main focus is on the investigation of properties of compression-molded films of poly(butylene 2,4-furanoate) (2,4-PBF) (Scheme 1). Besides a deep molecular, thermal, and diffractometric characterization, tensile and barrier properties of oxygen and carbon dioxide of both amorphous and semicrystalline films have been investigated and correlated to the film microstructure; this

Scheme 1. Poly(butylene 2,4-furanoate) (2,4-PBF) and Poly(butylene 2,5-furanoate) (2,5-PBF) Chemical Structures $^{a}$
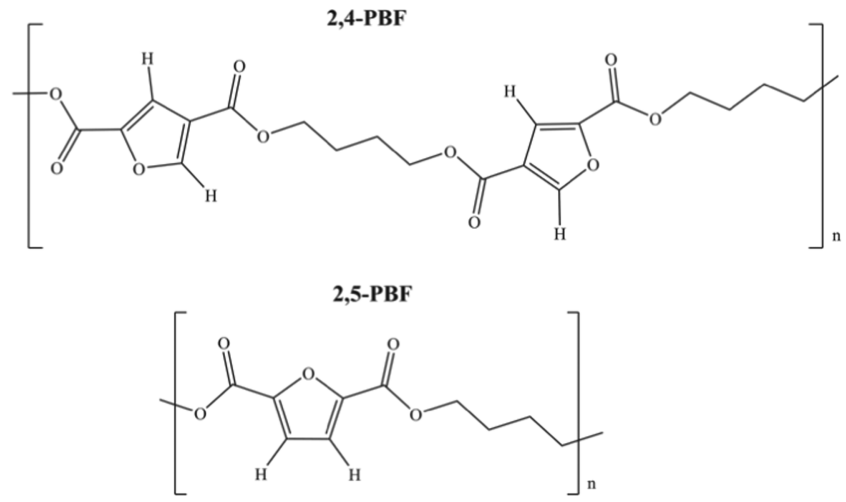

${ }^{a}$ Two repeating units with head-to-tail and tail-to-head orientations of a furan moiety are reported for 2,4-PBF. 
latter, in turn, is related to structural isomerism. 2,5-PBF (Scheme 1) has been also synthesized and processed in the form of a film for the sake of comparison.

\section{EXPERIMENTAL SECTION}

Materials. Dimethyl 2,4-furandicarboxylate (2,4-DMF) was synthesized according to the procedure reported previously. ${ }^{49}$ Furthermore, it was purified to the monomer grade by repeated recrystallization from methanol ( $2 \times$ times); 2,5-furandicarboxylic acid 98\% (2,5-FDCA) (Carbosynth Ltd., Compton, Berkshire, U.K.), 1,4-butanediol (BD), titanium tetrabutoxide (TBT), and titanium isopropoxide (TIP) (Sigma-Aldrich, Saint Louis, MO) were used as purchased.

2,5-Furandicarboxylic Acid Esterification. Esterification of 2,5-FDCA was carried out in a round-bottomed flask containing 25 $\mathrm{g}(0.160 \mathrm{~mol})$ of diacid and $390 \mathrm{~mL}(9.64 \mathrm{~mol})$ of methanol, a large molar excess with respect to the carboxylic acid terminals (about $1: 30)$. As in the procedure described in the literature, ${ }^{37}$ the suspension turned into a solution after heating at $70{ }^{\circ} \mathrm{C}$ for $30 \mathrm{~min}$ under magnetic stirring. The flask was then cooled down to room temperature, and then, $28 \mathrm{~mL}$ of thionyl chloride (1:1 molar ratio with respect to the $-\mathrm{COOH}$ groups) was added dropwise while keeping track of the temperature. Each drop originated a sizzling sound the moment it touched the liquid. The suspension was heated again at $70{ }^{\circ} \mathrm{C}$ under stirring for additional $3 \mathrm{~h}$, turning into a pale yellow solution. The flask was finally quenched in ice for $30 \mathrm{~min}$, making dimethyl furan-2,5-dicarboxylate (2,5-DMF) precipitate in the form of glossy white flakes. The product was filtered under vacuum and repeatedly washed with cold methanol. The obtained crystalline solid (22 g, $0.119 \mathrm{~mol}$, corresponding to a yield of $74 \%$ ) was dried overnight at room temperature and stored under vacuum before use.

Poly(butylene 2,4-furanoate) and Poly(butylene 2,5-furanoate) Synthesis. The synthesis of poly(butylene 2,4-furanoate) $(2,4-\mathrm{PBF})$ was carried out in a $250 \mathrm{~mL}$ stirred glass reactor put in a thermostated bath, according to the polycondensation conditions usually adopted for furan-based polyesters, ${ }^{37,44}$ starting from $2.8 \mathrm{~g}$ of dimethyl 2,4-furandicarboxylate (2,4-DMF), $2.9 \mathrm{~g}$ of 1,4-butanediol (1,4-BDO) (glycol $200 \mathrm{~mol} \%$ excess), and $200 \mathrm{ppm}$ of TIP used as the catalyst. The reaction was performed in the following two steps: the transesterification stage (first step) was carried out under a nitrogen flow for $2 \mathrm{~h}$ and the temperature was slowly increased from 180 to $200{ }^{\circ} \mathrm{C}$, while methanol was distilled off. The polymerization stage (second step) was carried out under vacuum to significantly increase the molecular weight of the product by eliminating the glycol excess. At $210{ }^{\circ} \mathrm{C}$, the pressure was lowered to $10 \mathrm{mbar}$ and then slowly decreased to 0.05 mbar over the course of $3 \mathrm{~h}$, showing a gradual increase of the torque value displayed by the stirrer up to a plateau.

Poly(butylene 2,5-furanoate) (2,5-PBF) was also synthesized using similar conditions as described for 2,4-PBF. The reagents used were $7 \mathrm{~g}$ of 2,5-DMF and $10 \mathrm{~g}$ of 1,4-BDO (glycol molar excess of about $300 \%$ ). In total, $200 \mathrm{ppm}$ of TBT and $200 \mathrm{ppm}$ of TIP were used as catalysts. The reaction conditions were previously described. ${ }^{37}$ The as-synthesized 2,4-PBF and 2,5-PBF homopolymers were purified by dissolution in hexafluoroisopropanol/chloroform $(5 \% \mathrm{v} / \mathrm{v})$ and precipitation in methanol.

Molecular Characterization. The chemical structure of the synthesized polymers was determined by means of proton- and carbon-nuclear magnetic resonance spectroscopy $\left({ }^{1} \mathrm{H}\right.$ NMR and ${ }^{13} \mathrm{C}$ NMR) using a Varian Inova 400-MHz (Agilent Technologies, Palo Alto, CA) at room temperature. Because of the different relaxation times of ${ }^{1} \mathrm{H}$ and ${ }^{13} \mathrm{C}$ atoms, about $10 \mathrm{mg}$ for ${ }^{1} \mathrm{H}$ NMR and $40 \mathrm{mg}$ for ${ }^{13} \mathrm{C}$ NMR, respectively, of all polymers were dissolved in $0.7 \mathrm{~mL}$ of deuterated chloroform (containing $0.03 \mathrm{vol} \%$ tetramethylsilane as an internal standard), with the help of a few droplets of trifluoroacetic acid added just before the measurements.

Gel permeation chromatography (GPC) was used to determine the molecular weight $\left(M_{n}\right)$ and the corresponding polydispersity index $(D)$ of the synthesized polymers using a 1525 binary HPLC pump (Waters, Milford, MA) equipped with a PLgel $5 \mathrm{~mm}$ MiniMIX-C column (Agilent Technologies) at $30{ }^{\circ} \mathrm{C}$. GPC-grade chloroform was used as an eluent, with a flow of $1 \mathrm{~mL} / \mathrm{min}$. The samples were prepared as polymer/chloroform solutions $(2 \mathrm{mg} / \mathrm{mL})$ with the addition of a few droplets of hexafluoroisopropanol. The calibration curve was obtained using polystyrene standards in the $550-2500000 \mathrm{~g} / \mathrm{mol}$ range.

Film Preparation. In total, $100 \mu \mathrm{m}$ thick free-standing films of 2,4-PBF and 2,5-PBF were prepared by compression molding using a C12 laboratory press (Carver, Wabash, IN). About $2.5 \mathrm{~g}$ of purified material was put in between two Teflon sheets, positioned in the press and heated to a temperature $40{ }^{\circ} \mathrm{C}$ higher than their melting temperature. After $1 \mathrm{~min}$, the pressure was increased to 9 ton $/ \mathrm{m}^{2}$ and maintained for $2 \mathrm{~min}$. Afterward, the films were quenched in iced water, obtaining amorphous specimens. Two additional films were prepared by cold crystallizing the initially amorphous compression-molded films (2,4-PBF, $75{ }^{\circ} \mathrm{C}$ for $17 \mathrm{~h}$; 2,5-PBF, $100{ }^{\circ} \mathrm{C}$ for $4 \mathrm{~h}$ ).

Thermal Characterization. TGA analyses were carried out on a TGA4000 (PerkinElmer, Waltham, MA), heating about $5 \mathrm{mg}$ of material at a constant rate $\left(10{ }^{\circ} \mathrm{C} / \mathrm{min}\right)$ in the temperature range of $40-800{ }^{\circ} \mathrm{C}$, under a flow of pure nitrogen $(40 \mathrm{~mL} / \mathrm{min}) . T_{5 \%}$ was calculated as the temperature corresponding to a weight loss of $5 \%$, $T_{\text {onset }}$ was calculated as the temperature corresponding to the beginning of weight loss, and $T_{\max }$ was calculated as the minimum value of the thermogram derivative. Thermal transitions were evaluated using a DSC6 (PerkinElmer, Waltham, MA). About $5 \mathrm{mg}$ of material was placed in an aluminum pan and subjected to the following thermal program: heating from -30 to $220{ }^{\circ} \mathrm{C}$ at $20{ }^{\circ} \mathrm{C} /$ min, cooling from 200 to $-30{ }^{\circ} \mathrm{C}$ at $100{ }^{\circ} \mathrm{C} / \mathrm{min}$, and heating from -30 to $220{ }^{\circ} \mathrm{C}$ at $20^{\circ} \mathrm{C} / \mathrm{min}$. The glass transition temperature $\left(\mathrm{T}_{\mathrm{g}}\right)$ was calculated as the midpoint of the glass-to-rubber transition step, while the specific heat increment $\left(\Delta C_{\mathrm{p}}\right)$ corresponds to the height between the two baselines related to the glass transition step. The melting temperature $\left(T_{\mathrm{m}}\right)$ and the cold crystallization temperature $\left(T_{\mathrm{cc}}\right)$ were determined as the peak maximum/minimum of the endothermic/exothermic phenomena in the DSC curve, respectively. The corresponding heat of fusion $\left(\Delta H_{\mathrm{m}}\right)$ and heat of crystallization $\left(\Delta H_{\mathrm{c}}\right)$ were obtained from the total area of the endothermic and exothermic signals, respectively.

Structural Characterization. Wide-angle X-ray scattering (WAXS) experiments were performed on film samples using Xrays from a copper source (wavelength of $0.154 \mathrm{~nm}$ ) on an X'PertPro diffractometer (PANalytical, Almelo, The Netherlands) equipped with a solid-state $X^{\prime}$ Celerator detector moving in $0.1^{\circ}$ steps, at a rate of $100 \mathrm{~s} / \mathrm{step}$. The indexes of crystallinity $\left(X_{\mathrm{c}}\right)$ were obtained from the X-ray diffraction profiles, calculating the ratio between the crystalline diffraction area $\left(A_{c}\right)$ and the total area of the diffraction profile $\left(A_{\mathrm{t}}\right) . A_{\mathrm{c}}$ was obtained by subtracting the amorphous halo from the total area of the diffraction profile. The incoherent scattering was excluded from these calculations.

Mechanical Characterization. Tensile tests were performed using an Instron 5966 (Instron, Norwood, MA) equipped with a rubber grip and a transducer-coupled $1 \mathrm{kN}$ load cell. Rectangular film samples $(5 \mathrm{~mm} \times 50 \mathrm{~mm}$, gauge length of $20 \mathrm{~mm})$ were stretched at a constant rate of $10 \mathrm{~mm} / \mathrm{min}$. The load-displacement results were converted into stress-strain curves, and the elastic modulus $(E)$ was calculated considering the initial linear slope. The results are reported as average value \pm standard deviation, obtained from at least five different tests for each material.

Water Contact Angle Test. Static contact angle measurements were performed on films of 2,4-PBF and 2,5-PBF with a DSA30S Drop Shape Analyzer (Kruss Scientific, Hamburg, Germany). A syringe was filled with deionized water at room temperature, and then, it was used to deposit a droplet on the film under observation. For each film, at least five drops were deposited on different areas and a picture of their profile was captured both just after deposition and $1 \mathrm{~min}$ after. The profiles were processed and contact angles were reported as the average value \pm standard deviation. 
Scheme 2. 2,4-PBF ${ }^{b}$

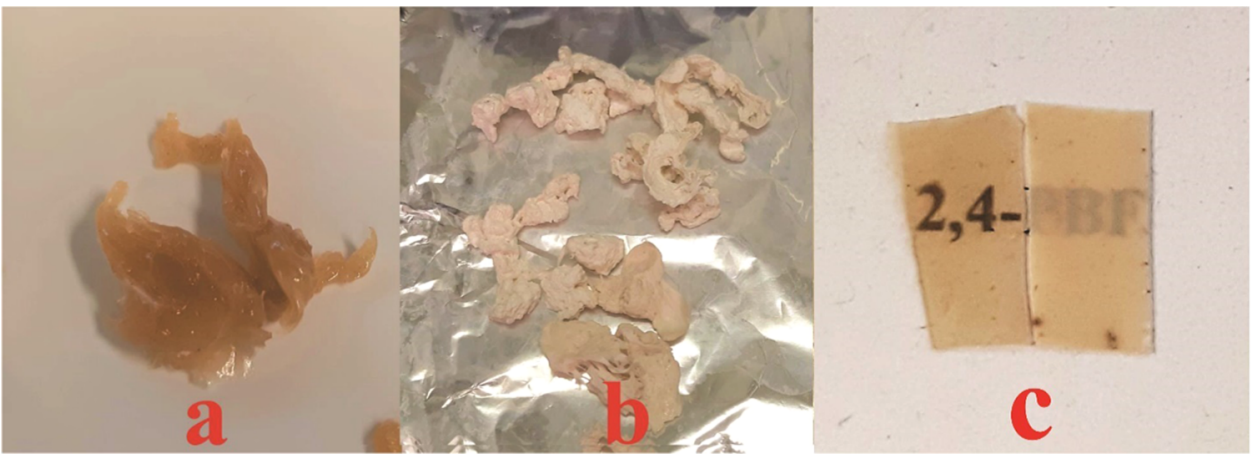

${ }^{b}$ (a) As-synthesized; (b) purified; and (c) left, amorphous film; right, annealed film.
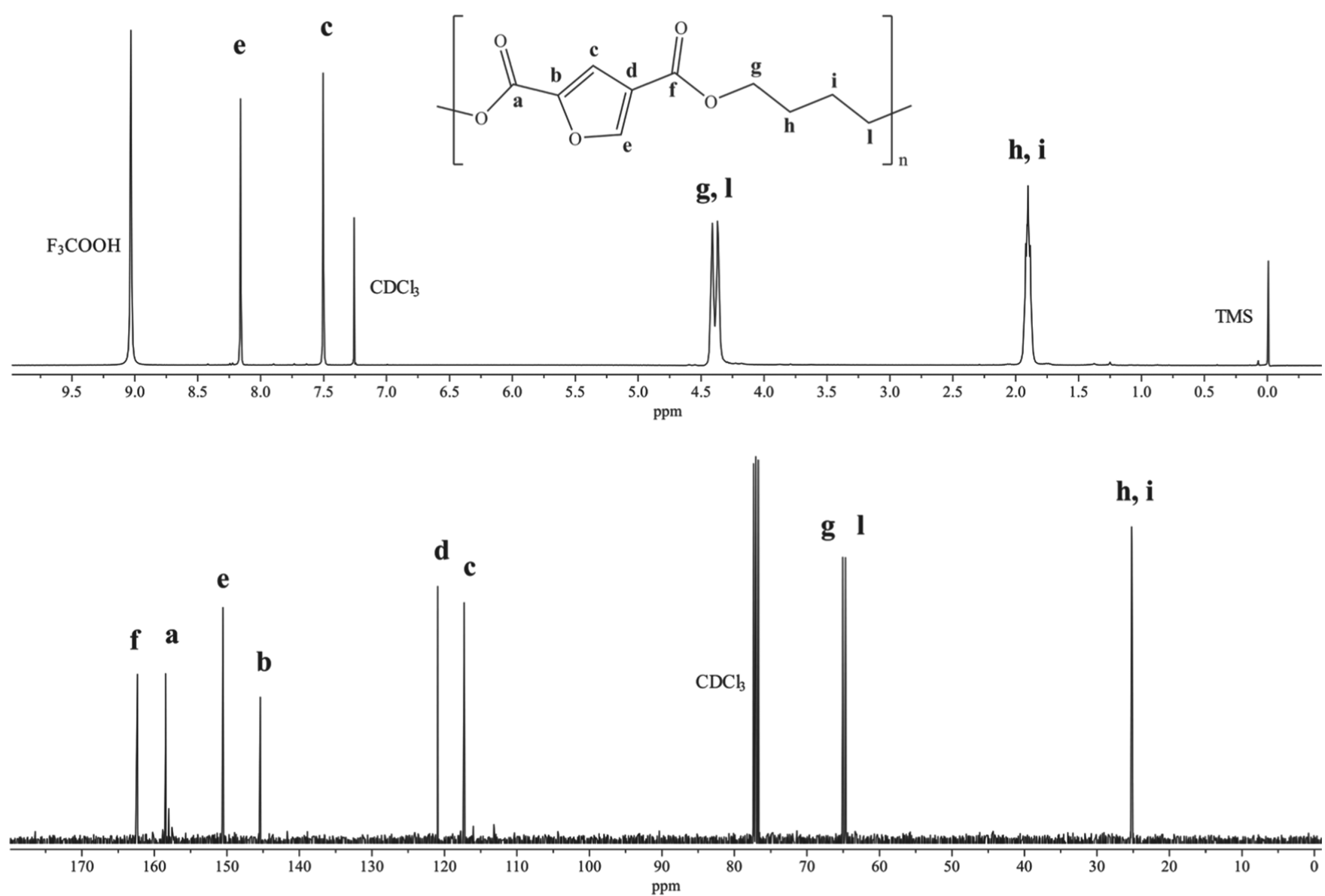

Figure 1. ${ }^{1} \mathrm{H}$ NMR (top) and ${ }^{13} \mathrm{C}$ NMR (bottom) spectra of the 2,4-PBF homopolymer with peak assignments.

Gas Barrier Property Evaluation. Barrier properties toward pure $\mathrm{O}_{2}$ and $\mathrm{CO}_{2}$ were tested through a manometric method using a permeance testing device, type GDP-C (Brugger Feinmechanik $\mathrm{GmbH}$, Munchen, Germany), according to the Gas Permeability Testing Manual and standards ASTM 1434-82 (standard test method for determining gas permeability characteristics of plastic film and sheeting, 2009), DIN 53536 (gas permeability standard), and ISO/DIS 15105-1 (plastic film and sheeting determination of gas transport rate; part I: differential pressure method, 2007). Each polymeric compression-molded film (diameter of $10 \mathrm{~cm}$, surface area of $78.5 \mathrm{~cm}^{2}$ ) was placed between two chambers, and the upper one was filled with the gas under investigation (pressure $=1 \mathrm{~atm}$, temperature $=23{ }^{\circ} \mathrm{C}$; gas stream $=100 \mathrm{~cm}^{3} / \mathrm{min}$; 0 or $85 \%$ relative humidity). In the lower chamber, a pressure transducer measured the increase in gas pressure as a function of time, and starting from the pressure-time plot, it was possible to calculate permeability and gas transmission rate (GTR) values, which represent the barrier properties of the film. The temperature was set by an external thermostat HAAKE-Circulator DC10-K15 type (ThermoFisher Scientific, Waltham, MA). Each measurement was performed in triplicate, reporting the mean value.

\section{RESULTS AND DISCUSSION}

Molecular Characterization. The as-synthesized 2,4-PBF homopolymer appeared as a light-colored solid with noteworthy tenacity to the touch (Scheme 2a). After the purification process described in the Experimental Section, it turned into white flocculates (Scheme 2b) that, by compression molding, produced a free-standing, yet flexible, 
Table 1. GPC, WAXS, DSC (I Scan, Unless Otherwise Stated), and TGA Data ${ }^{a}$

\begin{tabular}{|c|c|c|c|c|c|c|c|c|c|c|c|c|c|c|c|}
\hline & \multicolumn{2}{|c|}{ GPC } & \multicolumn{2}{|c|}{ WAXS } & \multicolumn{8}{|c|}{ DSC } & \multicolumn{3}{|c|}{ TGA } \\
\hline & $\begin{array}{c}M_{\mathrm{n}} \\
(\mathrm{g} / \mathrm{mol})\end{array}$ & $Ð$ & $X_{c}(\%)$ & $\begin{array}{l}\text { FWHM } \\
(\mathrm{deg})^{a}\end{array}$ & $\begin{array}{c}T_{g} \\
\left({ }^{\circ} \mathrm{C}\right)\end{array}$ & $\begin{array}{c}\Delta c_{\mathrm{p}} \\
\left(\mathrm{J} / \mathrm{g}^{\circ} \mathrm{C}\right)\end{array}$ & $\begin{array}{l}T_{\mathrm{cc}} \\
\left({ }^{\circ} \mathrm{C}\right)\end{array}$ & $\begin{array}{l}\Delta H_{\mathrm{cc}} \\
(\mathrm{J} / \mathrm{g})\end{array}$ & $\begin{array}{l}T_{\mathrm{m} 1} \\
\left({ }^{\circ} \mathrm{C}\right)\end{array}$ & $\begin{array}{l}\Delta H_{\mathrm{m} 1} \\
(\mathrm{~J} / \mathrm{g})\end{array}$ & $\begin{array}{l}T_{\mathrm{m} 2} \\
\left({ }^{\circ} \mathrm{C}\right)\end{array}$ & $\begin{array}{l}\Delta H_{\mathrm{m} 2} \\
(\mathrm{~J} / \mathrm{g})\end{array}$ & $\begin{array}{l}T_{5 \%} \\
\left({ }^{\circ} \mathrm{C}\right)\end{array}$ & $\begin{array}{l}T_{\text {onset }} \\
\left({ }^{\circ} \mathrm{C}\right)\end{array}$ & $\begin{array}{l}T_{\max } \\
\left({ }^{\circ} \mathrm{C}\right)\end{array}$ \\
\hline & & & & & & & 2,4-PBF & & & & & & & & \\
\hline purified & 44500 & 2.3 & $17 \pm 2$ & $0.8 ; 1.0$ & 33 & 0.185 & & & 74 & 1 & 106 & 9 & 378 & 396 & 409 \\
\hline $\begin{array}{l}\text { purified (II } \\
\text { scan) }\end{array}$ & & & 0 & & 33 & 0.337 & & & & & & & & & \\
\hline film & & & 0 & & 33 & 0.376 & & & & & & & & & \\
\hline annealed & & & $20 \pm 2$ & $0.6 ; 0.6$ & 33 & 0.290 & & & 80 & 10 & 106 & 13 & & & \\
\hline stretched & & & & & 33 & 0.285 & 57 & 8 & & & 113 & 16 & & & \\
\hline & & & & & & & 2,5-PBF & & & & & & & & \\
\hline purified & 32600 & 1.7 & $25 \pm 2$ & $1.7 ; 1.6$ & 40 & 0.110 & & & & & 164 & 33 & 369 & 390 & 407 \\
\hline $\begin{array}{l}\text { purified (II } \\
\text { scan) }\end{array}$ & & & 0 & & 39 & 0.318 & 107 & 25 & & & 165 & 25 & & & \\
\hline film & & & 0 & & 39 & 0.360 & 100 & 25 & & & 165 & 25 & & & \\
\hline annealed & & & $24 \pm 2$ & $1.3 ; 1.3$ & 41 & 0.111 & & & 127 & 8 & 164 & 28 & & & \\
\hline stretched & & & & & 42 & 0.269 & 55 & 16 & & & 167 & 35 & & & \\
\hline
\end{tabular}
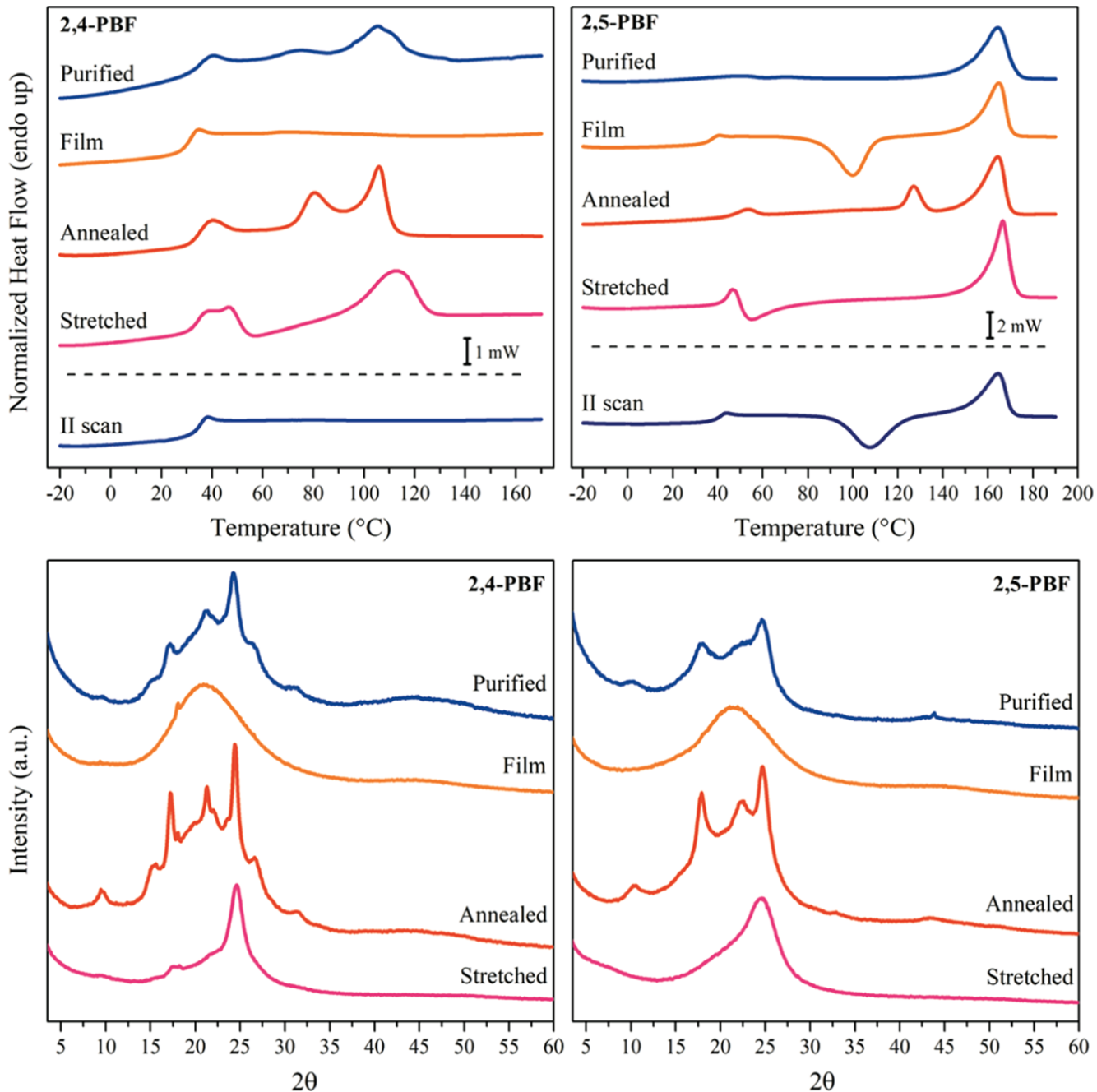

Figure 2. DSC traces (top row) and WAXS patterns (bottom row) of 2,4-PBF and 2,5-PBF homopolymers: purified samples (blue), compression-molded (orange), annealed (red), and stretched (pink) films. DSC heating from -20 to $200{ }^{\circ} \mathrm{C}$ at $20{ }^{\circ} \mathrm{C} / \mathrm{min}$ was applied to the different as-prepared samples (I scan) and to the melt-quenched one (II scan).

transparent light-colored film (Scheme $2 c$, on the left of the coin). The annealing process rendered the sample opaque and stiff, suggesting crystalline phase development (Scheme
$2 \mathrm{c}$, on the right of the coin). The analogous pictures for 2,5$\mathrm{PBF}$ are shown in Scheme S1. 
In Figure 1 , the ${ }^{1} \mathrm{H}$ NMR and ${ }^{13} \mathrm{C}$ NMR spectra of the 2,4PBF homopolymer are reported together with peak assignment. NMR analysis allowed confirming the 2,4-PBF chemical structure and excluding the occurrence of side reactions during the synthesis process since no additional signals were detected.

As concerns the ${ }^{1} \mathrm{H}$ NMR spectrum, in addition to the signals of the solvents $\left(\mathrm{CDCl}_{3}\right.$ and $\left.\mathrm{CF}_{3} \mathrm{COOH}\right)$ and the internal standard (tetramethylsilane, TMS), one can see the singlets at low field ascribable to the furan ring hydrogen atoms $\mathrm{e}$ and $\mathrm{c}$, at 8.16 and $7.50 \mathrm{ppm}$, respectively, while moving toward a higher field, the peaks of $\mathrm{g}$ and 1 protons (4.41 and $4.37 \mathrm{ppm}$ ) and the multiplet of $\mathrm{h}$ and $\mathrm{i}$ hydrogen atoms $(1.90 \mathrm{ppm})$ corresponding to the $-\mathrm{CH}_{2}-$ from $1,4-$ $\mathrm{BDO}$ are seen. As concerns the ${ }^{13} \mathrm{C}$ NMR spectrum: in the left region, one can find the peaks of the carboxylic group carbons, $f$ and a, at 162.3 and $158.4 \mathrm{ppm}$, followed by the $\mathrm{b}$, $\mathrm{d}$, e, and c carbon atoms of the furan ring at 145.4, 120.9, 150.5 , and $117.3 \mathrm{ppm}$, respectively. Finally, at lower ppm values, the signals of the glycol subunit were detected: the secondary carbons in $\alpha$-position to the carboxylic groups, $g$ and 1 , resonated very close to each other at 65.0 and 64.7 ppm, while the $\mathrm{h}$ and $\mathrm{i}$ methylene groups were located at $25.2 \mathrm{ppm}$.

The NMR analysis conducted on 2,5-PBF confirmed the chemical structure previously reported. ${ }^{37}$ The optimization of the synthesis process was also confirmed by GPC measurements. As one can see from the elugrams in Figure $\mathrm{S} 1$ and the data in Table 1, GPC analysis allowed determining the high molecular weight $\left(M_{n}\right)$ and narrow polydispersity index (Đ) for both PBF polymers. Even though 2,4-PBF showed higher $M_{\mathrm{n}}$, both polymers have a sufficiently high molecular weight; thus, the difference in $M_{n}$ and $Đ$ has not to be considered as an issue for the comparability of the functional properties of the two isomers. It is worth highlighting the compression molding process did not determine a $M_{n}$ decrease.

Thermal and Structural Characterization. Figure 2 reports the DSC traces of 2,4-PBF and 2,5-PBF homopolymers subjected to different thermal/processing treatments, with the corresponding data collected in Table 1 . As one can see, both the as-purified polymers show a calorimetric curve typical of semicrystalline materials being characterized by the endothermic step of the glass-to rubber transition at lower temperatures, followed by endothermic phenomena at higher temperatures, due to the isotropization of ordered phases. Nevertheless, the two isomers revealed differences both in position, multiplicity, and intensity of the melting peaks; in particular, 2,4-PBF showed double and less intense signals located at lower temperatures than the single melting peak of 2,5-PBF (Table 1).

The observed reductions of $T_{\mathrm{m}}\left(106\right.$ vs $\left.164{ }^{\circ} \mathrm{C}\right)$ and $\Delta H_{\mathrm{m}}$ ( $10 \mathrm{vs} 33 \mathrm{~J} / \mathrm{g}$ ) revealed for $2,4-\mathrm{PBF}$ are directly related to the low symmetry of the repeating unit and also of the macromolecular chain, this last due to a random distribution of the 2,4-furanic moiety, leading to head-to-tail and head-tohead (i.e., tail-to-tail) configurations. ${ }^{54}$

The different crystallization capability of the two homopolymers is also evidenced by the calorimetric traces of the compression-molded films (Figure 2). As a matter of fact, the DSC curve of the 2,4-PBF film showed just the $T_{\mathrm{g}}$ step at $33{ }^{\circ} \mathrm{C}$, indicating that the film was not able to organize in crystalline structures during the ballistic cooling of the melt to room temperature neither during the heating ramp. As concerns the 2,5-PBF isomer, a different behavior was revealed. As one can see from Figure 2 and the data in Table 1, once exceeded $T_{\mathrm{g}}$, the polymer chains can foldcrystallize at $100{ }^{\circ} \mathrm{C}\left(T_{\mathrm{cc}}\right)$ and finally melt at $165{ }^{\circ} \mathrm{C}$. Nevertheless, being $\Delta H_{\mathrm{cc}}=\Delta H_{\mathrm{m}}$, we can assume that even 2,5-PBF macromolecules were locked in the amorphous state during film preparation.

The amorphous 2,4-PBF and 2,5-PBF films were then submitted to (i) thermal annealing and (ii) mechanical stretching. The thermal response of the resulting specimens was checked, and the corresponding results are reported in Figure 2 and Table 1.

After permanence at $75{ }^{\circ} \mathrm{C}$ for $17 \mathrm{~h}$, the 2,4-PBF film showed a DSC trace similar to that of the purified sample but with more intense (Total $\Delta H_{\mathrm{m}}=23 \mathrm{~J} / \mathrm{g}$ ) and better-defined double melting peaks.

Also, in the case of the 2,5-PBF film, annealing $\left(100{ }^{\circ} \mathrm{C}\right.$ for $4 \mathrm{~h}$ ) determined polymer crystallization as evidenced by the double endothermic phenomena at 127 and $164{ }^{\circ} \mathrm{C}$ whose total melting heat, $\Delta H_{\mathrm{m}}$, resulted equal to $36 \mathrm{~J} / \mathrm{g}$. As wellknown, multiple melting signals can arise from the presence of different crystalline phases or from melting-crystallization-melting phenomena taking place during the heating scan. To shed light on this sense, wide-angle X-ray scattering analysis was carried out on all of the samples under study.

The calorimetric results obtained on the stretched films resulted very peculiarly. As one can see from Figure 2, the elongation of the compression-molded films produced monomodal melting peaks at temperatures higher than both purified and annealed samples. Furthermore, what might look like a tilted baseline of the stretched samples DSC trace in the $50-100{ }^{\circ} \mathrm{C}$ temperature range was interpreted as broad and asymmetric exotherms located just above the $T_{\mathrm{g}}$ step $\left(T_{\mathrm{cc}}\right.$ $=57$ and $55{ }^{\circ} \mathrm{C}$ for $2,4-\mathrm{PBF}$ and $2,5-\mathrm{PBF}$, respectively), whose associated energy is lower than the final melting enthalpy $\left(2,4-\mathrm{PBF}, \Delta H_{\mathrm{cc}}=8 \mathrm{~J} / \mathrm{g}\right.$ and $\Delta H_{\mathrm{m}}=16 \mathrm{~J} / \mathrm{g} ; 2,5-$ PBF, $\Delta H_{\mathrm{cc}}=16 \mathrm{~J} / \mathrm{g}$ and $\left.\Delta H_{\mathrm{m}}=35 \mathrm{~J} / \mathrm{g}\right)$. These results indicated that, for both polymers, elongation determined partial crystallization $\left(\Delta H_{\mathrm{c}}<\Delta H_{\mathrm{m}}\right)$, also promoting the orientation of macromolecules favoring their further organization in ordered structures during the subsequent DSC heating ramp. The diffractometric technique can be useful to investigate more in-depth the polymer chain orientation under stretching, too.

As concerns the glass-to-rubber transition temperature of the polymers under study, it was evaluated on the fully amorphous specimens obtained by fast cooling $\left(100{ }^{\circ} \mathrm{C} / \mathrm{min}\right)$ from the melt, performed to erase the previous thermal history. The corresponding II scans (Figure 2; Table 1) are very similar to the film I scan traces, evidencing that fast cooling is an effective tool to get both polymers amorphous. The $\mathrm{T}_{\mathrm{g}}$ values so determined turned out to be 33 and $39{ }^{\circ} \mathrm{C}$ for 2,4-PBF and 2,5-PBF, respectively. As well known, the glass-to-rubber transition temperature mainly depends on polymer free volume, directly related to the chemical formula and also configuration, i.e., spatial arrangement, as well as on the interchain interactions. All of these parameters affect chain mobility. As expected, considering that the two polymers under study are structural isomers, the two $T_{\mathrm{g}}$ 's are very close to each other. Nevertheless, the different furan ring configurations could induce changes in both the free volume and interchain interaction. The sum of all of the just 
mentioned parameters determines higher mobility for 2,4PBF (lower $T_{\mathrm{g}}$ ) compared to that for 2,5-PBF (higher $T_{\mathrm{g}}$ ).

Since thermal stability is a key parameter for material processing, both $\mathrm{PBF}$ polymers have been subjected to thermogravimetric analysis (TGA) under an inert atmosphere. The curves and the relative onset temperature $\left(T_{\text {onset }}\right)$ and the temperatures corresponding to $5 \%$ weight loss and maximum degradation rate $\left(T_{5 \%}\right.$ and $\left.T_{\max }\right)$ are reported in Figure 3 and Table 1, respectively. First, it is worth

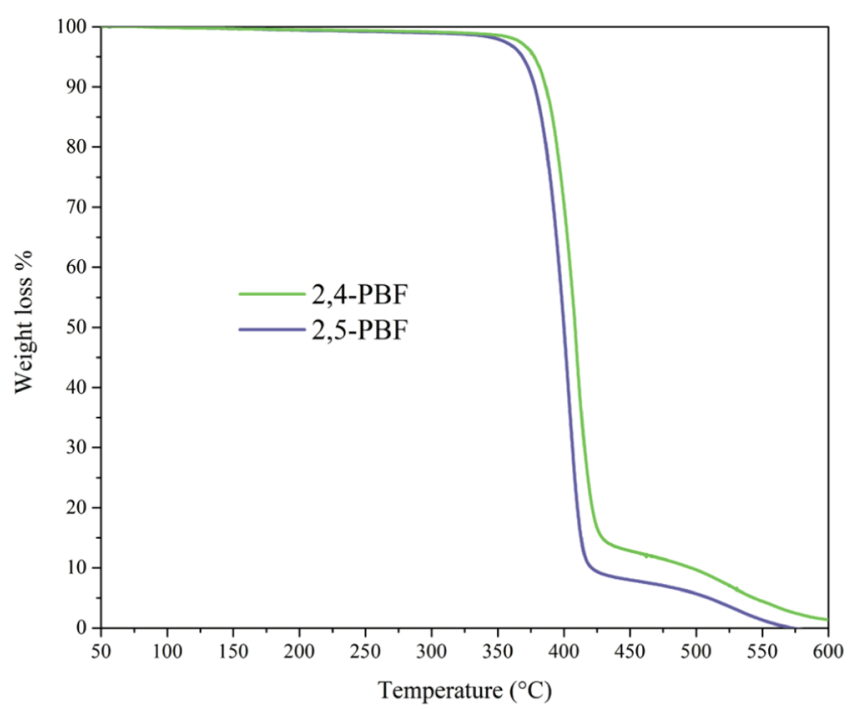

Figure 3. TGA curves of 2,4-PBF and 2,5-PBF homopolymers acquired by heating the polymers from 50 to $800{ }^{\circ} \mathrm{C}$ at $10{ }^{\circ} \mathrm{C} / \mathrm{min}$ under nitrogen flow $(40 \mathrm{~mL} / \mathrm{min})$.

highlighting the impressive thermal stability of both isomers, in particular of 2,4-PBF. Since the two materials are characterized by the same furan ring and carboxylic group density and aliphatic segment length, the higher stability of 2,4-PBF could be due to the higher aromaticity of the furan subunit coming from the different position of the two -COOR groups on the ring and/or to a different balance of interchain interactions as already reported for poly(pentamethylene 2,5-furanoate) (2,5-PPeF). ${ }^{37}$ The slightly higher $M_{\mathrm{n}}$ value determined for 2,4-PBF could also improve its thermal stability. The TGA results obtained are comparable to the ones previously reported for semicrystalline 2,5-PBF. ${ }^{37}$

Samples were submitted to WAXS investigation to get deeper details on structural features. Figure 2 shows the WAXS patterns of 2,4-PBF and 2,5-PBF samples prepared in different conditions; the relative crystallinity degree values $\left(X_{c}\right)$ and peak widths (FWHM) are reported in Table 1 . The purified 2,4-PBF sample in the form of powder exhibited seven peaks at 2 -theta values (interplanar distances in $\AA$ are reported in parentheses) of $9.7^{\circ}(9.1), 15.5^{\circ}(5.7), 17.3^{\circ}$ (5.1), $21.3^{\circ}$ (4.2), $24.4^{\circ}(3.6), 26.7^{\circ}$ (3.3), and $31.3^{\circ}(2.9)$. The compression-molded film showed only the bell-shaped background, typical of an amorphous material. The very tiny peak at $18^{\circ}$ for the 2,4-PBF film (barely detectable also in the annealed and stretched samples) is due to inorganic contaminants, present in a negligible amount. The annealed film displayed Bragg reflections at the same angular values and with relative intensities comparable to the purified powder one but with sharper and better-resolved peaks. This is good evidence that they contain the same crystal phase, although the crystallinity is higher as expected in the light of calorimetric results. Moreover, the crystal domains are bigger in the annealed sample since an inverse relationship exists between the peak width (FWHM) and the size of crystallite domains. The profile of the film sample previously stretched, collected in the equatorial projection, showed only two broad peaks at 17.4 and $24.4^{\circ}$ overlapped to an amorphous halo, suggesting the possible presence of a mesophase i.e., a phase with a poor three-dimensional order. A molecular reorganization from a partial order assembly up to get a crystal form can be the origin of the exothermic event with $\Delta H_{\mathrm{cc}}<\Delta H_{\mathrm{m}}$ observed after $T_{\mathrm{g}}$ in the DSC scan. The pattern of the 2,4PBF stretched film is similar to that one described for a sample analogously treated of poly(butylene 2,5-thiophenedicarboxylate) (PBTF), although the peak positions are slightly different. $^{56}$

Purified 2,5-PBF powder is semicrystalline, with peaks at $10.4^{\circ}(d=8.5 \AA), 17.7^{\circ}(5.0)$, and $24.7^{\circ}$ (3.6) overlapped to a bell-shaped background, while the film is completely amorphous. Also, in the case of 2,5-PBF, after annealing, the film showed improved crystallinity. The pattern resembled the powder one, but a further peak is evident at $22.3^{\circ}(d=4.0 \AA)$. The pattern detected in powder and film 2,5-PBF samples indicated the presence of the crystal phase previously reported by $\mathrm{Zhu}$ et al. for 2,5-PBF. ${ }^{10}$ The scan collected after stretching the sample displays a bell-shaped background with the addition of one very broad and intense peak that can be due to the poor ordered phase. An overall comparison between the patterns of the two isomers suggested that 2,4-PBF samples, with sharper peaks (FWHM in Table 1), have bigger crystal domains. 2,5-PBF samples show higher values of crystallinity $\left(X_{c}\right.$ in Table 1$)$, which means the volume fraction arranged in the tridimensional order is bigger. A comparison between patterns of 2,4$\mathrm{PBF}$ and 2,5-PBF annealed films revealed the reflections of $2,4-\mathrm{PBF}$ are in greater number and, the most intense, are displaced by -0.3 and $-0.7^{\circ}$ with respect to those of 2,5 PBF. For these reasons, although in the two series the molecular unit is very similar, it is plausible that the crystal phase of the two isomers is different. Not even the comparison with the three crystal phases reported for $\mathrm{PBTF}^{56}$ allows to establish a possible similarity with the 2,4-PBF structure.

Functional Property Characterization. The stressstrain curves are given in Figure S3, and the obtained results are presented in Table 2 . The most representative curves are reported in Figure 4. The amorphous 2,4-PBF film is characterized by the excellent mechanical response, i.e., quite high elastic modulus $(E)$ and stress at break $\left(\sigma_{\mathrm{b}}\right)$, accompanied by outstanding elongation at break $\left(\varepsilon_{\mathrm{b}}\right)$, all of these parameters contributing to remarkable toughness. The

Table 2. Mechanical Characterization Data

\begin{tabular}{|c|c|c|c|c|}
\hline & \multicolumn{2}{|c|}{ 2,4-PBF } & \multicolumn{2}{|c|}{ 2,5-PBF } \\
\hline & amorphous & annealed & amorphous & annealed \\
\hline$E(\mathrm{MPa})$ & $939 \pm 72$ & $1330 \pm 180$ & $1307 \pm 34$ & $1382 \pm 125$ \\
\hline$\sigma_{\mathrm{y}}(\mathrm{MPa})$ & $16 \pm 1$ & $35 \pm 5$ & $42 \pm 6$ & \\
\hline$\varepsilon_{\mathrm{y}}(\%)$ & $3.7 \pm 0.3$ & $4.8 \pm 1.1$ & $3.8 \pm 0.8$ & \\
\hline$\sigma_{\mathrm{b}}(\mathrm{MPa})$ & $16 \pm 6$ & $24 \pm 1$ & $24 \pm 5$ & $56 \pm 6$ \\
\hline$\varepsilon_{\mathrm{b}}(\%)$ & $564 \pm 139$ & $208 \pm 82$ & $312 \pm 61$ & $6.2 \pm 0.5$ \\
\hline
\end{tabular}




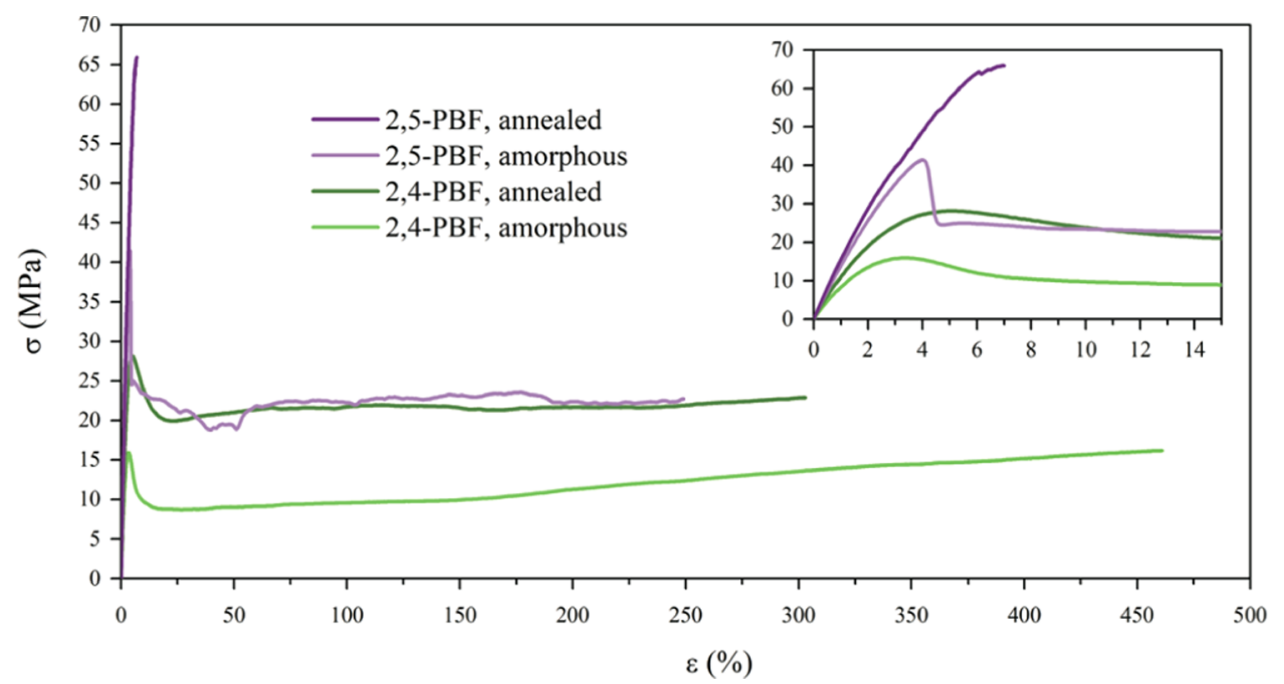

Figure 4. Stress-strain curves of the amorphous (light-colored) and annealed (dark-colored) 2,4-PBF (green) and 2,5-PBF (purple) films. Inset: magnification at low elongation.

annealing process permitted to further enhance the mechanical resistance ( $E$ and $\sigma_{\mathrm{b}}$ increased) thanks to the development of crystalline microstructure, as evidenced by DSC analysis, without affecting significantly the $\varepsilon_{\mathrm{b}}$ value that remained quite high. Comparing the two PBFs, it is important to note that the value of strain at break could be influenced by the difference in molecular weights of the polymers, contributing to a higher strain at break in the case of 2,4-PBF. In any case, one can see that the amorphous 2,5PBF sample presented even better mechanical resistance (higher $E$ and $\sigma_{\mathrm{b}}$ ) but lower $\varepsilon_{\mathrm{b}}$, in line with the lower molecular mobility, i.e., greater $T_{\mathrm{g}}$ value (Table 1 ). It can be also deducted from the experimental results that the amorphous 2,5-PBF film presented tensile behavior similar to that of annealed 2,4-PBF, in terms of $E, \sigma_{\mathrm{b}}$, and $\varepsilon_{\mathrm{b}}$. Nevertheless, annealed 2,4-PBF is supposed to maintain its functional properties up to $70{ }^{\circ} \mathrm{C}$, a temperature at which it starts melting, while amorphous $2,5-\mathrm{PBF}$ is stable below its $T_{\mathrm{g}}\left(39^{\circ} \mathrm{C}\right)$. It can be noted that in the case of amorphous 2,5-PBF, the shape of the curve in Figure 4 is irregular (like various others shown in Figure S3); this evolution could be due to the changes in the geometry of the sample being stretched, as well as to microstructure developed during the test as confirmed by the calorimetric results for the stretched samples (Table 1 and Figure 2). The development of crystals in the 2,5-PBF film determined a further increase of elastic modulus and stress but also an important decrement of elongation at break. These results are comparable to the ones previously reported for semicrystalline 2,5-PBF. ${ }^{37}$ Finally, the stress-strain curves for all of the films investigated showed a yielding point at quite low stress and elongation values $\left(\sigma_{y}\right.$ and $\varepsilon_{y}$ ), which lead to excluding the elastic behavior of the materials under investigation. The yielding phenomena shown in Figure 4 are associated with the stress and strain conditions at which the sliding of macromolecular chains becomes irreversible, i.e., the initially elastic deformation turns plastic. Yielding can be evidenced both in the amorphous and semicrystalline samples, except for annealed 2,5-PBF, probably because it easily breaks under stress.

Water contact angle (WCA) measurements were carried out on both amorphous and annealed 2,4-PBF and 2,5-PBF films. The water drop profile was measured just after deposition and also after $1 \mathrm{~min}$. The calculated WCA values are collected in Table 3 .

Table 3. Water Contact Angle Values of the Just Deposited Drop $\left(\mathrm{WCA}_{0}\right)$ and After $1 \mathrm{~min}\left(\mathrm{WCA}_{1}\right)^{a}$

\begin{tabular}{llllll} 
& \multicolumn{2}{c}{$2,4-\mathrm{PBF}$} & & \multicolumn{2}{c}{$2,5-\mathrm{PBF}$} \\
\cline { 2 - 3 } \cline { 5 - 6 } & amorphous & annealed & & amorphous & annealed \\
$\mathrm{WCA}_{0}$ & $98.1 \pm 1.8$ & $97.1 \pm 3.7$ & & $95.2 \pm 2.4$ & $90.5 \pm 1.8$ \\
$\mathrm{WCA}_{1}$ & $98.1 \pm 1.8$ & $91.3 \pm 3.1$ & & $90.2 \pm 2.2$ & $85.9 \pm 1.4$ \\
$\mathrm{WCA}_{0}-\mathrm{WCA}_{1}$ & 0 & 5.8 & 5 & 4.6
\end{tabular}

${ }^{a}$ Units are expressed in degrees.

As an example, in Figure 5, the drop shape on the amorphous films, at $0 \mathrm{~s}$ and $1 \mathrm{~min}$ from deposition, is also

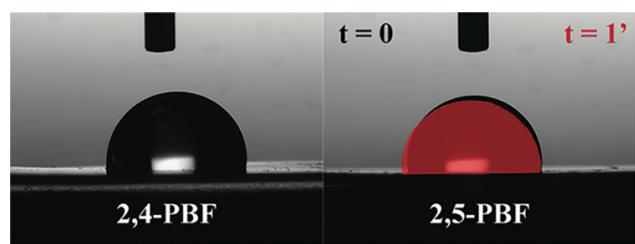

Figure 5. Water drop profiles on amorphous 2,4-PBF and 2,5-PBF surfaces. Black profiles refer to the just deposited drop, while the red one refers to the drop after $1 \mathrm{~min}$ from deposition.

reported. As one can see from the profiles in Figure 5 and the results in Table 3, both the amorphous $\mathrm{PBF}$ isomers show quite high WCA values, in particular 2,4-PBF.

The deposited water drop, as described in Scheme 3, left, simultaneously interacts with the polar subunits (-COOgroups and furan rings) and with the nonpolar moieties (methylene groups), showing modest affinity with polymer films. Nevertheless, a slight difference in WCA values evidenced higher hydrophilicity for the 2,5-PBF film (lower WCA), in line with the results reported by Bourdet et al., 52 who provided evidence that 2,5-PEF has a higher average dipole moment than 2,4-PEF. In fact, even if both isomers have the same density of electric dipoles (coming from $-\mathrm{COO}-$ groups and furan rings), the average dipole 
Scheme 3. (Left Panel) Representation of Polymer Chain Interactions with Water Drop (•) and $\mathrm{CO}_{2} \mathrm{Molecules}^{(\bullet)}$ and (Right panel) Representation of $\mathrm{H}$ Bonds (red dashed line) in 2,5-PBF and 2,4-PBF
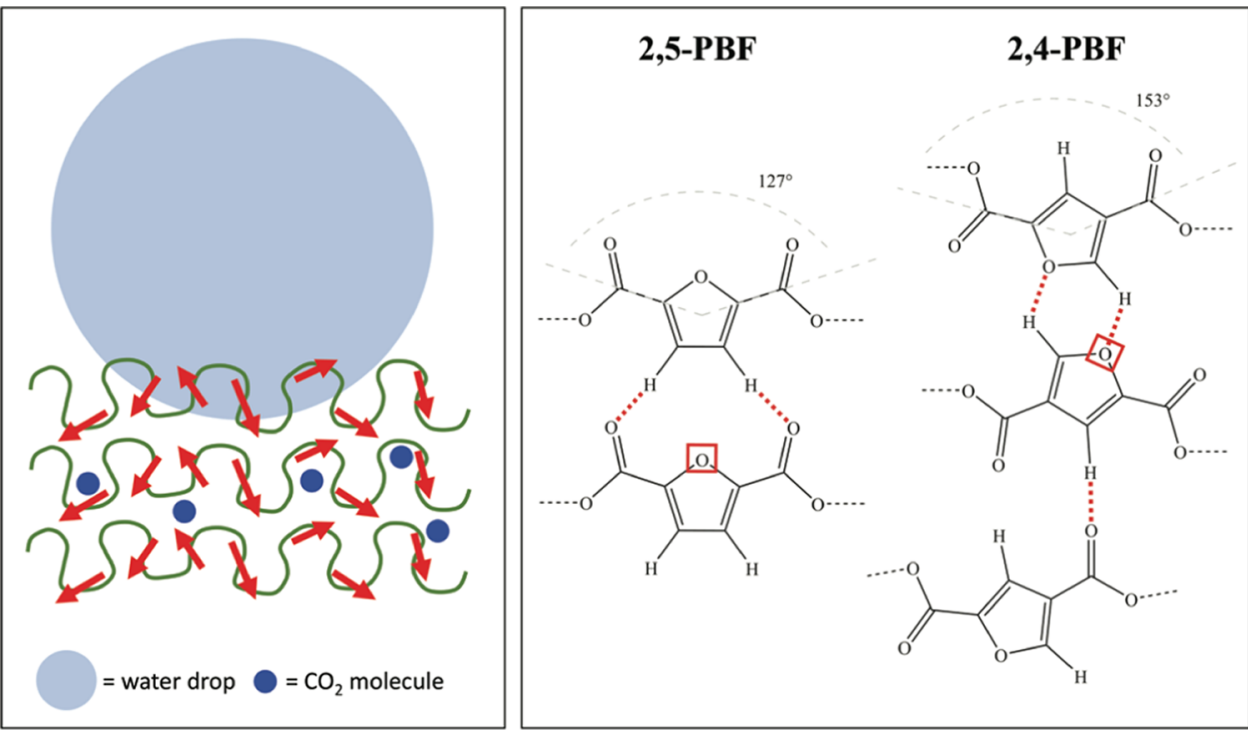

${ }^{c}$ Green segments represent the nonpolar subunits (mainly glycolic moieties), while the red arrows indicate the electric dipoles coming from $-\mathrm{COO}-$ groups as well as furan rings. Ester link angles are from ref 54.

Table 4. $\mathrm{O}_{2}$ and $\mathrm{CO}_{2}$ Transmission Rates for the Compression-Molded Amorphous and Annealed 2,4-PBF and 2,5-PBF Films at $23{ }^{\circ} \mathrm{C}$ Both in Dry (0\% Relative Humidity, 0\% RH) and Humid (85\% RH) Atmospheres

\begin{tabular}{|c|c|c|c|c|c|}
\hline & & \multicolumn{2}{|c|}{ 2,4-PBF } & \multicolumn{2}{|c|}{ 2,5-PBF } \\
\hline & & amorphous & annealed & amorphous & annealed \\
\hline \multirow[t]{2}{*}{$\mathrm{O}_{2}-\mathrm{TR}\left(\mathrm{cm}^{3} \mathrm{~cm}\right) /\left(\mathrm{m}^{2} \mathrm{~d} \mathrm{~atm}\right)$} & $0 \% \mathrm{RH}$ & 0.0022 & 0.131 & 0.104 & 0.149 \\
\hline & $85 \% \mathrm{RH}$ & 0.0026 & 0.125 & 0.0914 & 0.172 \\
\hline \multirow{2}{*}{$\mathrm{CO}_{2}-\mathrm{TR}\left(\mathrm{cm}^{3} \mathrm{~cm}\right) /\left(\mathrm{m}^{2} \mathrm{~d}\right.$ atm $)$} & $0 \% \mathrm{RH}$ & 0.0007 & 0.0580 & 0.0800 & 0.227 \\
\hline & $85 \% \mathrm{RH}$ & 0.0021 & 0.0822 & 0.0950 & 0.176 \\
\hline
\end{tabular}
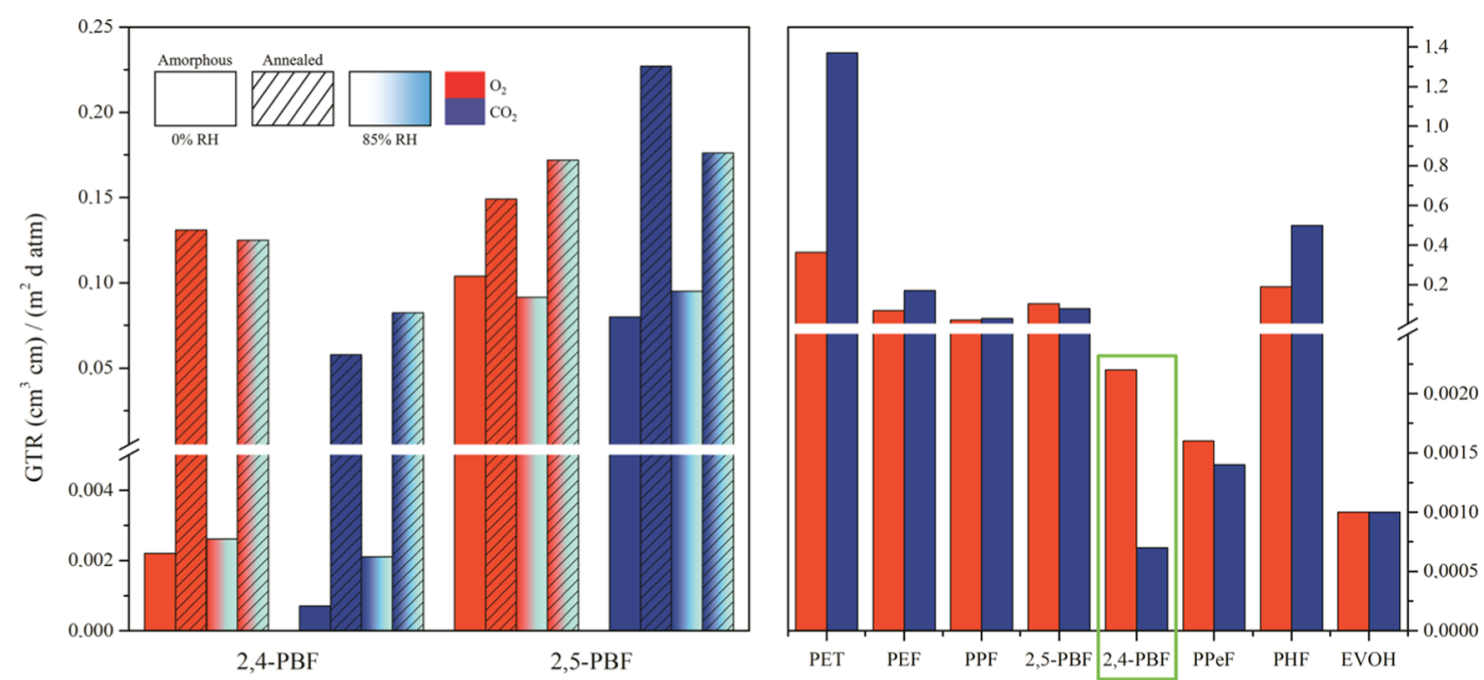

Figure 6. Gas transmission rates to $\mathrm{O}_{2}$ and $\mathrm{CO}_{2}$ at $23{ }^{\circ} \mathrm{C}$ : (left panel) for amorphous and annealed 2,4-PBF and 2,5-PBF both in dry (0\% RH) and humid $(85 \% \mathrm{RH})$ atmospheres and (right panel) 2,4-PBF and 2,5-PBF GTR values at $23{ }^{\circ} \mathrm{C}$ and $0 \% \mathrm{RH}$ also compared with EVOH (32\% ethylene), ${ }^{63} \mathrm{PET},{ }^{62} \mathrm{PEF},{ }^{11,23} \mathrm{PPF}^{37} \mathrm{PPeF}^{37}$ and poly(hexamethylene 2,5-furanoate) (PHF). ${ }^{37}$

moment, strongly dependent on the polar group steric orientation, turns out to be lower for the 2,4-isomer.

Considering the annealed films, a decrease of the WCA value could be evidenced for the 2,5-PBF sample. To explain this result, one should take into account that the development of the crystalline phase (as suggested by DSC and
WAXS data) produces a roughness surface increase that, in turn, can enhance the hydrophilic character, i.e., WCA value reduction. ${ }^{57} \mathrm{~A}$ decrease of around $5^{\circ}$ of the water contact angle was also recorded with time for the annealed 2,4-PBF and the amorphous and annealed 2,5-PBF films (see Figure 5 and Table 3). It can be supposed that, with time, the water- 
polar segment interactions prevail over water-apolar subunit ones. No time effect was detected for the most hydrophobic sample, amorphous 2,4-PBF.

The permeability performance to two different pure gases, $\mathrm{O}_{2}$ and $\mathrm{CO}_{2}$, was evaluated on the 2,4-PBF and 2,5-PBF compression-molded films, both amorphous and annealed, at $23{ }^{\circ} \mathrm{C}$ under dry $(0 \% \mathrm{RH})$ and wet $(85 \% \mathrm{RH})$ atmospheres. The so determined results, expressed as the gas transmission rate (GTR), are collected in Table 4 and Figure 6 (the raw data is reported in the SI). In the case of semicrystalline 2,5$\mathrm{PBF}$, the results obtained are in perfect accordance with the barrier properties previously determined by Guidotti et al. ${ }^{37}$

From the conducted measurements, the effect of isomerism, relative humidity, and the presence of ordered phases can be inferred.

The influence of the different atom connections, i.e., structural isomerism, can be evaluated considering the two amorphous 2,4-PBF and 2,5-PBF films tested at $23{ }^{\circ} \mathrm{C}$ and dry conditions.

As one can see from the GTR values reported in Table 4 and Figure 6, the 2,4-PBF isomer showed really outstanding barrier properties to both $\mathrm{O}_{2}$ and $\mathrm{CO}_{2}$, much better than the 2,5-PBF ones, evidencing the deep effect of tiny chemical modification on the final functional response like gas barrier capability. The contribution of the higher molecular weight of 2,4-PBF should be limited considering the improvement up to two orders of magnitude in GRT values for the 2,4-isomer. More in detail, the $\mathrm{O}_{2}$-TR and $\mathrm{CO}_{2}$-TR values for the 2,4isomer are 50 and 110 times lower, respectively, than the 2,5isomer ones. These surprising results cannot be explained just on the basis of macromolecular chain mobility and free volume, related to the glass transition temperature, being $T_{\mathrm{g} \text { 2,4-PBF }}<T_{\mathrm{g} 2,5-\mathrm{PBF}}$ (see Figure 2 and Table 1). In fact, the lower the $T_{g}$, the higher the number of unoccupied spaces, which should cause an increase of the gas transmission rate (GTR), contrary to what is observed experimentally. An explanation should be sought by considering the establishment of intermolecular interactions. Previous studies conducted on aromatic polymers containing phenyl and thiophene moieties have evidenced the formation of interplanar $\pi-\pi$ stacking of the rings, possibly originating a mesophase from which a more ordered 3D structure could develop. $^{58-60}$ In addition to this kind of intermolecular interplay, another type of noncovalent interaction has been described in the literature for aromatic furan-based polyesters. $^{33,37,52}$ Both simulation ${ }^{52}$ and experimental ${ }^{33,37}$ studies evidenced the presence of interchain side-by-side hydrogen bonds, leading to a pretty compact macromolecular array able to very efficiently lock the gas molecule passage through the polymer matrix. In the case of the 2,5-PBF isomer, considering the geometric features of the 2,5-furan ring, in particular the angle in between the $-\mathrm{COO}-$ moieties (as described in Scheme 3, right), it can be supposed that the interchain $\mathrm{H} \cdots \mathrm{O}$ bonds mainly involve the ring hydrogen atoms and the carboxylic oxygen ones. On the other hand, the ring $\mathrm{O}$ atom becomes hardly accessible because of the steric hindrance, limiting its involvement in the formation of hydrogen bonds. In this sense, the different position of the oxygen in the 2,4-furan ring makes it much more sterically accessible, enhancing its capability to form hydrogen bonds (Scheme 3, right). Consequently, a much more compact structure can develop in 2,4-PBF, coming from a higher $\mathrm{H}$ - bond density, involving both the ring and carboxylic $\mathrm{O}$ atoms.

Very interestingly, the perm-selectivity ratio, defined as $\mathrm{GTR}_{\mathrm{CO}_{2}} / \mathrm{GTR}_{\mathrm{O}_{2}}$, that was reported to be $<1$ for 2,5-furanbased polymers ${ }^{33,37}$ thanks to the good $\mathrm{CO}_{2}$ solubility in the polar polymer matrix, remains favorable and even lower for the 2,4-PBF isomer, regardless of its lower polarity evidenced by WCA tests. This unexpected datum can be explained considering the very local $\mathrm{CO}_{2}$ molecule-polymer chain interaction. Carbon dioxide, in fact, can efficiently diffuse in the polymer volume adopting the best conformation to enhance local dipole-dipole interactions (Scheme 3, left).

The presence of hydrogen bonds in both 2,4-PBF and 2,5PBF films is also corroborated by their performance in a humid atmosphere $(85 \% \mathrm{RH})$. As well known, ${ }^{61}$ water molecules typically exert a plasticizing effect, worsening the final gas barrier performance, unless hydrogen bonds are present in the polymer film. In this last case, $\mathrm{H}_{2} \mathrm{O}$ molecules actively interact with polymer macromolecules, enhancing the $\mathrm{H}$-bond bridges, thus further improving gas locking and, consequently, reducing GTR values (Figure 6 and Table 4).

Another quite surprising result regards the semicrystalline films. In fact, after ordered structures had developed by annealing, the GTR values to both oxygen and carbon dioxide increase. This experimental evidence suggests that, even if more compact microstructures able to stop the $\mathrm{O}_{2}$ and $\mathrm{CO}_{2}$ molecules formed in the two PBF films, the disclination content also increases, creating interphase spaces through which gas molecules can more easily pass. The higher the initial amorphous film performance, the greater the crystallinity incidence in terms of barrier property detriment.

Amorphous 2,4-PBF gas performance, under dry conditions and at $23{ }^{\circ} \mathrm{C}$, was also compared with the commercially used poly(ethylene terephthalate) (PET) and the most known poly(ethylene furanoate) (PEF), 11,23,62 with other furan-based polyesters synthesized and characterized by us ${ }^{33,37}$ and with ethylene vinyl alcohol tested by other authors. ${ }^{63}$ As one can see from Figure 6, right, 2,4-PBF showed the best gas properties together with 2,5-PPeF. It is worth highlighting that, with respect to 2,5-PPeF, 2,4-PBF presents better mechanical resistance (higher elastic modulus and stress to strain). 2,4-PBF gas barrier response is also comparable to the ethylene vinyl alcohol $(\mathrm{EVOH})$ sample $^{63}$ but with much better water resistance.

\section{CONCLUSIONS}

In the present work, for the first time, a 2,4-FDCA-based polyester has been successfully processed by compression molding into a free-standing flexible film of $11 \mathrm{~cm}$ diameter. Such a result is proof that the solvent-free polymerization process used for the synthesis of poly(butylene 2,4-furanoate) (2,4-PBF) has produced a high-molecular-weight polymer. Film mechanical response and gas barrier performances were investigated to evaluate the potential applicability of the new material in sustainable food packaging.

First, a comparison with the 2,5-PBF isomer showed that isomerism is a key parameter in determining the final properties of the material. In fact, significant differences between the two isomers have been found. Specifically, compared to 2,5-PBF, 2,4-PBF is characterized by

- higher thermal stability, a crucial property during polymer processing, which associated with a lower $T_{\mathrm{g}}$ 
in the case of the amorphous sample and a lower $T_{\mathrm{m}}$ for semicrystalline one determines a wider processability window of the material;

- lower ability to crystallize with consequent easier production of transparent films, required in food packaging;

- lower hydrophilicity, which means higher polymer resistance to humidity;

- superior mechanical properties, as the amorphous sample is tough; through a proper annealing process, mechanical resistance can be further improved, and mechanical response become equivalent to that of amorphous 2,5-PBF, but with the advantage of preserving its properties up to a higher temperature;

- superior gas barrier properties (to oxygen and carbon dioxide) in the amorphous form, with a particularly significant improvement in the case of $\mathrm{CO}_{2}$ gas, which could potentially make this polymer suitable for the production of bottles for soft drinks.

It is also important to underline how 2,4-PBF shows significantly better barrier performance than 2,5-PEF, which is currently considered the most credible biobased substitute of PET. Compared to poly(pentamethylene 2,5-furanoate) recently investigated and characterized by exceptional gas barrier performance similar to EVOH, 2,4-PBF presents comparable gas barrier response, maintained in humid conditions, but higher mechanical resistance.

In conclusion, thanks to the combination of all of the above-mentioned characteristics, 2,4-PBF represent a further very important member of the furan-based polyester family, opening up new possibilities in sustainable packaging applications.

\section{ASSOCIATED CONTENT}

\section{SI Supporting Information}

The Supporting Information is available free of charge at https://pubs.acs.org/doi/10.1021/acssuschemeng.1c04104.

2,5-PBF (Scheme S1), results of the ${ }^{1} \mathrm{H}$ NMR analysis (Table S1), ${ }^{1} \mathrm{H}$ NMR spectra of dimethyl 2,5furandicarboxylate (Figure $\mathrm{S} 1$ ), results of the GPC analysis on 2,4-PBF and 2,5-PBF (Figure S2), stressstrain curves of amorphous and annealed 2,5-PBF and 2,4-PBF (Figure S3), and $\mathrm{dP}$ vs time graph on amorphous 2,5-PBF and GTR vs time graph on amorphous (Figures S4-S35) (PDF)

\section{AUTHOR INFORMATION}

\section{Corresponding Authors}

Shanmugam Thiyagarajan - Wageningen Food \& Biobased Research, 6700 AA Wageningen, The Netherlands;

(1) orcid.org/0000-0002-7905-4233;

Email: shanmugam.thiyagarajan@wur.nl

Nadia Lotti - Civil, Chemical, Environmental and Materials Engineering Department, University of Bologna, 40131 Bologna, Italy; Interdepartmental Center for Industrial Research on Advanced Applications in Mechanical Engineering and Materials Technology, CIRI-MAM and Interdepartmental Center for Agro-Food Research, CIRIAGRO, University of Bologna, Bologna 40126, Italy; (1) orcid.org/0000-0002-7976-2934; Email: nadia.lotti@ unibo.it

\section{Authors}

Enrico Bianchi - Civil, Chemical, Environmental and Materials Engineering Department, University of Bologna, 40131 Bologna, Italy

Michelina Soccio - Civil, Chemical, Environmental and Materials Engineering Department, University of Bologna, 40131 Bologna, Italy; Interdepartmental Center for Industrial Research on Advanced Applications in Mechanical Engineering and Materials Technology, CIRIMAM, University of Bologna, Bologna 40126, Italy; (1) orcid.org/0000-0003-3646-9612

Valentina Siracusa - Department of Chemical Science, University of Catania, Catania 95125, Italy

Massimo Gazzano - Institute of Organic Synthesis and Photoreactivity, ISOF-CNR, 40129 Bologna, Italy

Complete contact information is available at: https://pubs.acs.org/10.1021/acssuschemeng.1c04104

\section{Author Contributions}

E.B. and M.S. contributed equally to this work. E.B. performed polymer synthesis, characterization, data curation, and visualization. M.S. supervised the experimental activity, analyzed the overall experimental data, wrote the original draft, and corrected and revised the manuscript. V.S. performed gas barrier measurements and corresponding data analysis and correction and revision of the manuscript. M.G. performed X-ray diffraction measurements and data analysis and correction and revision of the manuscript. N.L. supervised the experimental activity, analyzed the overall experimental data, wrote the original draft, corrected and revised the manuscript, and conceptualized and supervised the work and research funding. S.T. performed monomer synthesis and characterization, conceptualized and supervised the work and research funding, and corrected and revised the manuscript.

Notes

The authors declare no competing financial interest.

\section{ACKNOWLEDGMENTS}

E.B., M.S., and N.L. acknowledge the Italian Ministry of University and Research. This publication is based on the work from COST Action FUR4Sustain, CA18220, supported by COST (European Cooperation in Science and Technology).

\section{REFERENCES}

(1) Geyer, R.; Jambeck, J. R.; Law, K. L. Production, Use, and Fate of All Plastics Ever Made. Sci. Adv. 2017, 3, No. e1700782.

(2) Hahladakis, J. N.; Velis, C. A.; Weber, R.; Iacovidou, E.; Purnell, P. An Overview of Chemical Additives Present in Plastics: Migration, Release, Fate and Environmental Impact during Their Use, Disposal and Recycling. J. Hazard. Mater. 2018, 344, 179-199. (3) Schnurr, R. E. J.; Alboiu, V.; Chaudhary, M.; Corbett, R. A.; Quanz, M. E.; Sankar, K.; Srain, H. S.; Thavarajah, V.; Xanthos, D.; Walker, T. R. Reducing Marine Pollution from Single-Use Plastics (SUPs): A Review. Mar. Pollut. Bull. 2018, 137, 157-171.

(4) Xanthos, D.; Walker, T. R. International Policies to Reduce Plastic Marine Pollution from Single-Use Plastics (Plastic Bags and Microbeads): A Review. Mar. Pollut. Bull. 2017, 118, 17-26.

(5) Niaounakis, M. Recycling of Biopolymers - The Patent Perspective. Eur. Polym. J. 2019, 114, 464-475.

(6) Vollmer, I.; Jenks, M. J. F.; Roelands, M. C. P.; White, R. J.; van Harmelen, T.; de Wild, P.; van der Laan, G. P.; Meirer, F.; Keurentjes, J. T. F.; Weckhuysen, B. M. Beyond Mechanical 
Recycling: Giving New Life to Plastic Waste. Angew. Chem. Int. Ed. 2020, 59, 15402-15423.

(7) Soares, M. J.; Dannecker, P.-K.; Vilela, C.; Bastos, J.; Meier, M. A. R.; Sousa, A. F. Poly(1,20-Eicosanediyl 2,5-Furandicarboxylate), a Biodegradable Polyester from Renewable Resources. Eur. Polym. J. 2017, 90, 301-311.

(8) Tsanaktsis, V.; Papageorgiou, G. Z.; Bikiaris, D. N. A Facile Method to Synthesize High-Molecular-Weight Biobased Polyesters from 2,5-Furandicarboxylic Acid and Long-Chain Diols. J. Polym. Sci. A Polym. Chem. 2015, 53, 2617-2632.

(9) Tsanaktsis, V.; Terzopoulou, Z.; Nerantzaki, M.; Papageorgiou, G. Z.; Bikiaris, D. N. New Poly(Pentylene Furanoate) and Poly(Heptylene Furanoate) Sustainable Polyesters from Diols with Odd Methylene Groups. Mater. Lett. 2016, 178, 64-67.

(10) Zhu, J.; Cai, J.; Xie, W.; Chen, P. H.; Gazzano, M.; Scandola, M.; Gross, R. A. Poly(Butylene 2,5-Furan Dicarboxylate), a Biobased Alternative to PBT: Synthesis, Physical Properties, and Crystal Structure. Macromolecules 2013, 46, 796-804.

(11) Papamokos, G.; Dimitriadis, T.; Bikiaris, D. N.; Papageorgiou, G. Z.; Floudas, G. Chain Conformation, Molecular Dynamics, and Thermal Properties of Poly(n-Methylene 2,5-Furanoates) as a Function of Methylene Unit Sequence Length. Macromolecules 2019, 52, 6533-6546.

(12) Haernvall, K.; Zitzenbacher, S.; Amer, H.; Zumstein, M. T.; Sander, M.; McNeill, K.; Yamamoto, M.; Schick, M. B.; Ribitsch, D.; Guebitz, G. M. Polyol Structure Influences Enzymatic Hydrolysis of Bio-Based 2,5-Furandicarboxylic Acid (FDCA) Polyesters. Biotechnol. J. 2017, 12, No. 1600741.

(13) Fehrenbacher, U.; Grosshardt, O.; Kowollik, K.; Tübke, B.; Dingenouts, N.; Wilhelm, M. Synthese Und Charakterisierung von Polyestern Und Polyamiden Auf Der Basis von Furan-2,5Dicarbonsäure. Chem. Ing. Tech. 2009, 81, 1829-1835.

(14) Jiang, M.; Liu, Q.; Zhang, Q.; Ye, C.; Zhou, G. A Series of Furan-Aromatic Polyesters Synthesized via Direct Esterification Method Based on Renewable Resources. J. Polym. Sci. A Polym. Chem. 2012, 50, 1026-1036.

(15) Gomes, M.; Gandini, A.; Silvestre, A. J. D.; Reis, B. Synthesis and Characterization of Poly(2,5-Furan Dicarboxylate)s Based on a Variety of Diols. J. Polym. Sci. A Polym. Chem. 2011, 49, 3759-3768.

(16) Hu, H.; Zhang, R.; Shi, L.; Ying, W. B.; Wang, J.; Zhu, J. Modification of Poly(Butylene 2,5-Furandicarboxylate) with Lactic Acid for Biodegradable Copolyesters with Good Mechanical and Barrier Properties. Ind. Eng. Chem. Res. 2018, 57, 11020-11030.

(17) Fei, X.; Wang, J.; Zhu, J.; Wang, X.; Liu, X. Biobased Poly(Ethylene 2,5-Furancoate): No Longer an Alternative, but an Irreplaceable Polyester in the Polymer Industry. ACS Sustainable Chem. Eng. 2020, 8, 8471-8485.

(18) Knoop, R. J. I.; Vogelzang, W.; van Haveren, J.; van Es, D. S. High Molecular Weight Poly(Ethylene-2,5-Furanoate); Critical Aspects in Synthesis and Mechanical Property Determination. J. Polym. Sci. A Polym. Chem. 2013, 51, 4191-4199.

(19) Burgess, S. K.; Leisen, J. E.; Kraftschik, B. E.; Mubarak, C. R.; Kriegel, R. M.; Koros, W. J. Chain Mobility, Thermal, and Mechanical Properties of Poly(Ethylene Furanoate) Compared to Poly(Ethylene Terephthalate). Macromolecules 2014, 47, 13831391.

(20) Wang, J.-G.; Liu, X.-Q.; Zhu, J. From Furan to High Quality Bio-Based Poly(Ethylene Furandicarboxylate). Chin. J. Polym. Sci. 2018, 36, 720-727.

(21) Burgess, S. K.; Karvan, O.; Johnson, J. R.; Kriegel, R. M.; Koros, W. J. Oxygen Sorption and Transport in Amorphous Poly(Ethylene Furanoate). Polymer 2014, 55, 4748-4756.

(22) Burgess, S. K.; Mikkilineni, D. S.; Yu, D. B.; Kim, D. J.; Mubarak, C. R.; Kriegel, R. M.; Koros, W. J. Water Sorption in Poly(Ethylene Furanoate) Compared to Poly(Ethylene Terephthalate). Part 2: Kinetic Sorption. Polymer 2014, 55, 6870-6882.

(23) Burgess, S. K.; Kriegel, R. M.; Koros, W. J. Carbon Dioxide Sorption and Transport in Amorphous Poly(Ethylene Furanoate). Macromolecules 2015, 48, 2184-2193.
(24) Davidson, M. G.; Elgie, S.; Parsons, S.; Young, T. J. Production of HMF, FDCA and Their Derived Products: A Review of Life Cycle Assessment (LCA) and Techno-Economic Analysis (TEA) Studies. Green Chem. 2021, 23, 3154-3171.

(25) Rajesh, R. O.; Godan, T. K.; Sindhu, R.; Pandey, A.; Binod, P. Bioengineering Advancements, Innovations and Challenges on Green Synthesis of 2, 5-Furan Dicarboxylic Acid. Bioengineered 2020, 11, 19-38.

(26) Hu, H.; Zhang, R.; Wang, J.; Ying, W. B.; Shi, L.; Yao, C.; Kong, Z.; Wang, K.; Zhu, J. A Mild Method to Prepare High Molecular Weight Poly(Butylene Furandicarboxylate-Co-Glycolate) Copolyesters: Effects of the Glycolate Content on Thermal, Mechanical, and Barrier Properties and Biodegradability. Green Chem. 2019, 21, 3013-3022.

(27) Xie, H.; Wu, L.; Li, B.-G.; Dubois, P. Modification of Poly(Ethylene 2,5-Furandicarboxylate) with Biobased 1,5-Pentanediol: Significantly Toughened Copolyesters Retaining High Tensile Strength and O2 Barrier Property. Biomacromolecules 2019, 20, 353-364.

(28) Wang, J.; Liu, X.; Jia, Z.; Liu, Y.; Sun, L.; Zhu, J. Synthesis of Bio-Based Poly(Ethylene 2,5-Furandicarboxylate) Copolyesters: Higher Glass Transition Temperature, Better Transparency, and Good Barrier Properties. J. Polym. Sci. A Polym. Chem. 2017, 55, 3298-3307.

(29) Zhang, Q.; Song, M.; Xu, Y.; Wang, W.; Wang, Z.; Zhang, L. Bio-Based Polyesters: Recent Progress and Future Prospects. Prog. Polym. Sci. 2021, 120, No. 101430.

(30) Jiang, Y.; Woortman, A. J. J.; Alberda van Ekenstein, G. O. R.; Loos, K. A Biocatalytic Approach towards Sustainable FuranicAliphatic Polyesters. Polym. Chem. 2015, 6, 5198-5211.

(31) Guidotti, G.; Soccio, M.; Lotti, N.; Gazzano, M.; Siracusa, V.; Munari, A. Poly(Propylene 2,5-Thiophenedicarboxylate) vs. Poly(Propylene 2,5-Furandicarboxylate): Two Examples of High Gas Barrier Bio-Based Polyesters. Polymers 2018, 10, No. 785.

(32) Genovese, L.; Soccio, M.; Lotti, N.; Munari, A.; Szymczyk, A.; Paszkiewicz, S.; Linares, A.; Nogales, A.; Ezquerra, T. A. Effect of Chemical Structure on the Subglass Relaxation Dynamics of Biobased Polyesters as Revealed by Dielectric Spectroscopy: 2,5Furandicarboxylic Acid vs. Trans-1,4-Cyclohexanedicarboxylic Acid. Phys. Chem. Chem. Phys. 2018, 20, 15696-15706.

(33) Guidotti, G.; Soccio, M.; García-Gutiérrez, M. C.; GutiérrezFernández, E.; Ezquerra, T. A.; Siracusa, V.; Munari, A.; Lotti, N. Evidence of a 2D-Ordered Structure in Biobased Poly(Pentamethylene Furanoate) Responsible for Its Outstanding Barrier and Mechanical Properties. ACS Sustainable Chem. Eng. 2019, 7, 17863-17871.

(34) Guidotti, G.; Genovese, L.; Soccio, M.; Gigli, M.; Munari, A.; Siracusa, V.; Lotti, N. Block Copolyesters Containing 2,5-Furan and Trans-1,4-Cyclohexane Subunits with Outstanding Gas Barrier Properties. Int. J. Mol. Sci. 2019, 20, No. 2187.

(35) Soccio, M.; Costa, M.; Lotti, N.; Gazzano, M.; Siracusa, V.; Salatelli, E.; Manaresi, P.; Munari, A. Novel Fully Biobased Poly(Butylene 2,5-Furanoate/Diglycolate) Copolymers Containing Ether Linkages: Structure-Property Relationships. Eur. Polym. J. 2016, 81, 397-412.

(36) Guidotti, G.; Soccio, M.; Lotti, N.; Siracusa, V.; Gazzano, M.; Munari, A. New Multi-Block Copolyester of 2,5-Furandicarboxylic Acid Containing PEG-like Sequences to Form Flexible and Degradable Films for Sustainable Packaging. Polym. Degrad. Stab. 2019, 169, No. 108963.

(37) Guidotti, G.; Soccio, M.; García-Gutiérrez, M. C.; Ezquerra, T.; Siracusa, V.; Gutiérrez-Fernández, E.; Munari, A.; Lotti, N. Fully Biobased Superpolymers of 2,5-Furandicarboxylic Acid with Different Functional Properties: From Rigid to Flexible, High Performant Packaging Materials. ACS Sustainable Chem. Eng. 2020, 8, 95589568.

(38) Soccio, M.; Martínez-Tong, D. E.; Guidotti, G.; RoblesHernández, B.; Munari, A.; Lotti, N.; Alegria, A. Broadband 
Dielectric Spectroscopy Study of Biobased Poly(Alkylene 2,5Furanoate)s' Molecular Dynamics. Polymers 2020, 12, 1355.

(39) Robles-Hernández, B.; Soccio, M.; Castrillo, I.; Guidotti, G.; Lotti, N.; Alegria, A.; Martínez-Tong, D. E. Poly(Alkylene 2,5Furanoate)s Thin Films: Morphology, Crystallinity and Nanomechanical Properties. Polymer 2020, 204, No. 122825.

(40) Guidotti, G.; Mart, D. E.; Soccio, M.; Robles-Hernandez, B.; Gazzano, M.; Lotti, N.; Alegria, A. Evidence of Nanostructure Development from the Molecular Dynamics of Poly (Pentamethylene 2, 5-Furanoate). Macromolecules 2020, 53, 10526-10537.

(41) Sousa, A. F.; Vilela, C.; Fonseca, A. C.; Matos, M.; Freire, C. S. R.; Gruter, G. J. M.; Coelho, J. F. J.; Silvestre, A. J. D. Biobased Polyesters and Other Polymers from 2,5-Furandicarboxylic Acid: A Tribute to Furan Excellency. Polym. Chem. 2015, 6, 5961-5983.

(42) Soccio, M.; Lotti, N.; Munari, A.; Rebollar, E.; MartínezTong, D. E. Wrinkling Poly(Trimethylene 2,5-Furanoate) FreeStanding Films: Nanostructure Formation and Physical Properties. Polymer 2020, 202, No. 122666.

(43) Carlos Morales-Huerta, J.; Martínez de Ilarduya, A.; MuñozGuerra, S. Poly(Alkylene 2,5-Furandicarboxylate)s (PEF and PBF) by Ring Opening Polymerization. Polymer 2016, 87, 148-158.

(44) Papageorgiou, G. Z.; Papageorgiou, D. G.; Terzopoulou, Z.; Bikiaris, D. N. Production of Bio-Based 2,5-Furan Dicarboxylate Polyesters: Recent Progress and Critical Aspects in Their Synthesis and Thermal Properties. Eur. Polym. J. 2016, 83, 202-229.

(45) Terzopoulou, Z.; Tsanaktsis, V.; Nerantzaki, M.; Papageorgiou, G. Z.; Bikiaris, D. N. Decomposition Mechanism of Polyesters Based on 2,5-Furandicarboxylic Acid and Aliphatic Diols with Medium and Long Chain Methylene Groups. Polym. Degrad. Stab. 2016, 132, 127-136.

(46) Papageorgiou, D. G.; Guigo, N.; Tsanaktsis, V.; Exarhopoulos, S.; Bikiaris, D. N.; Sbirrazzuoli, N.; Papageorgiou, G. Z. Fast Crystallization and Melting Behavior of a Long-Spaced Aliphatic Furandicarboxylate Biobased Polyester, Poly(Dodecylene 2,5-Furanoate). Ind. Eng. Chem. Res. 2016, 55, 5315-5326.

(47) Genovese, L.; Lotti, N.; Siracusa, V.; Munari, A. Poly(Neopentyl Glycol Furanoate): A Member of the Furan-Based Polyester Family with Smart Barrier Performances for Sustainable Food Packaging Applications. Materials 2017, 10, No. 1028.

(48) Soccio, M.; Martínez-Tong, D. E.; Alegría, A.; Munari, A.; Lotti, N. Molecular Dynamics of Fully Biobased Poly(Butylene 2,5Furanoate) as Revealed by Broadband Dielectric Spectroscopy. Polymer 2017, 128, 24-30.

(49) Thiyagarajan, S.; Pukin, A.; Van Haveren, J.; Lutz, M.; Van Es, D. S. Concurrent Formation of Furan-2,5- and Furan-2,4Dicarboxylic Acid: Unexpected Aspects of the Henkel Reaction. RSC Adv. 2013, 3, 15678-15686.

(50) Thiyagarajan, S.; Vogelzang, W.; J I Knoop, R.; Frissen, A. E.; Van Haveren, J.; Van Es, D. S. Biobased Furandicarboxylic Acids (FDCAs): Effects of Isomeric Substitution on Polyester Synthesis and Properties. Green Chem. 2014, 16, 1957-1966.

(51) Fosse, C.; Bourdet, A.; Ernault, E.; Esposito, A.; Delpouve, N.; Delbreilh, L.; Thiyagarajan, S.; Knoop, R. J. I.; Dargent, E. Determination of the Equilibrium Enthalpy of Melting of Two-Phase Semi-Crystalline Polymers by Fast Scanning Calorimetry. Thermochim. Acta 2019, 677, 67-78.

(52) Bourdet, A.; Esposito, A.; Thiyagarajan, S.; Delbreilh, L.; Affouard, F.; Knoop, R. J. I.; Dargent, E. Molecular Mobility in Amorphous Biobased Poly(Ethylene 2,5-Furandicarboxylate) and Poly(Ethylene 2,4-Furandicarboxylate). Macromolecules 2018, 51, 1937-1945.

(53) Thiyagarajan, S.; Meijlink, M. A.; Bourdet, A.; Vogelzang, W.; Knoop, R. J. I.; Esposito, A.; Dargent, E.; Van Es, D. S.; Van Haveren, J. Synthesis and Thermal Properties of Bio-Based Copolyesters from the Mixtures of 2,5- A Nd 2,4-Furandicarboxylic Acid with Different Diols. ACS Sustainable Chem. Eng. 2019, 7, 18505-18516.

(54) Nolasco, M. M.; Araujo, C. F.; Thiyagarajan, S.; Rudić, S.; Vaz, P. D.; Silvestre, A. J. D.; Ribeiro-Claro, P. J. A.; Sousa, A. F.
Asymmetric Monomer, Amorphous Polymer? Structure-Property Relationships in 2,4-FDCA and 2,4-PEF. Macromolecules 2020, 53, $1380-1387$.

(55) Zaidi, S.; Thiyagarajan, S.; Bougarech, A.; Sebti, F.; Abid, S.; Majdi, A.; Silvestre, A. J. D.; Sousa, A. F. Highly Transparent Films of New Copolyesters Derived from Terephthalic and 2,4Furandicarboxylic Acids. Polym. Chem. 2019, 10, 5324-5332.

(56) Guidotti, G.; Gigli, M.; Soccio, M.; Lotti, N.; Gazzano, M.; Siracusa, V.; Munari, A. Ordered Structures of Poly(Butylene 2,5Thiophenedicarboxylate) and Their Impact on Material Functional Properties. Eur. Polym. J. 2018, 106, 284-290.

(57) Lin, F. Y. H.; Li, D.; Neumann, A. W. Effect of Surface Roughness on the Dependence of Contact Angles on Drop Size. J. Colloid Interface Sci. 1993, 159, 86-95.

(58) Sago, T.; Itagaki, H.; Asano, T. Onset of Forming Ordering in Uniaxially Stretched Poly(Ethylene Terephthalate) Films Due to $\pi-\pi$ Interaction Clarified by the Fluorescence Technique. Macromolecules 2014, 47, 217-226.

(59) Kaloni, T. P.; Giesbrecht, P. K.; Schreckenbach, G.; Freund, M. S. Polythiophene: From Fundamental Perspectives to Applications. Chem. Mater. 2017, 29, 10248-10283.

(60) Rudyak, V. Y.; Gavrilov, A. A.; Guseva, D. V.; Tung, S.-H.; Komarov, P. V. Accounting for $\pi-\pi$ Stacking Interactions in the Mesoscopic Models of Conjugated Polymers. Mol. Syst. Des. Eng. 2020, 5, 1137-1146.

(61) Hedenqvist, M. S. Barrier Packaging Materials, 2nd ed.; Elsevier, 2012.

(62) Hu, Y. S.; Prattipati, V.; Mehta, S.; Schiraldi, D. A.; Hiltner, A.; Baer, E. Improving Gas Barrier of PET by Blending with Aromatic Polyamides. Polymer 2005, 46, 2685-2698.

(63) McKeen, L. W. Polyolefins, Polyvinyls, and Acrylics. In Permeability Properties of Plastics and Elastomers; Elsevier, 2012; pp 145-193. 